Edmundo Arteaga-Fernández

\title{
Cardiomiopatia hipertrófica: estudo da sobrevida e de fatores prognósticos
}

Tese apresentada à Faculdade de Medicina da Universidade de São Paulo para obtenção do título de Doutor em Medicina

Área de concentração: Cardiologia

Orientador: Prof. Dr. Charles Mady

São Paulo

1998 


\section{Resumo}

Arteaga-Fernández E. Cardiomiopatia hipertrófica: estudo da sobrevida e de fatores prognósticos [tese]. São Paulo: Faculdade de Medicina, Universidade de São Paulo, 1998.

Com o objetivo de avaliar a sobrevida e os fatores prognósticos da cardiomiopatia hipertrófica estudamos, de forma prospectiva, 214 pacientes matriculados no ambulatório de Cardiopatias Gerais do Instituto do Coração do Hospital das Clínicas da Faculdade de Medicina da Universidade de São Paulo, entre 1980 e 1997. A idade

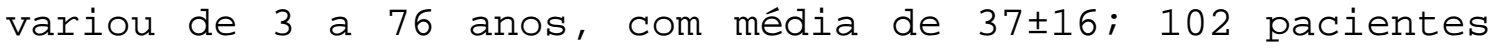
eram do sexo masculino e 112 do sexo feminino. Os pacientes foram submetidos a eletrocardiografia, eletrocardiografia dinâmica de 24 horas, ecocardiografia e estudo hemodinâmico. A média do tempo de seguimento foi de $88 \pm 56$ meses com variação de 13 a 299. Observamos 22 óbitos, sendo 14 (6\%) relacionados à doença e destes, 11/14 (78\%) faleceram subitamente. A probabilidade de sobrevida em cinco anos foi de 93,8\%, 87,7\% em dez anos e 76,4\% em 15 anos. A taxa de mortalidade anual foi de 0,4\%. A análise estatística univariada de cada uma das 23 variáveis clínicas e das 20 obtidas pelos exames complementares mostrou que apenas a forma familiar foi fator de risco de óbito. As variáveis forma familiar, idade menor que 20 anos, síncope e classe funcional foram selecionadas para análise multivariada pelo modelo de Cox e método stepwise. Novamente, a forma familiar foi identificada como fator . de risco independente de óbito. Podemos concluir, com base em nossos dados de casuística de centro de referência que, a longo prazo, a sobrevida dos pacientes portadores de cardiomiopatia hipertrófica foi benigna e que a forma familiar é fator de risco de óbito. 


\section{Summary}

Arteaga-Fernández E. Hypertrophic cardiomyopathy: study of survival and prognostic factors [tese]. São Paulo:

Faculdade de Medicina, Universidade de São Paulo, 1998.

To evaluate the prognostic factors related to long-term survival in hypertrophic cardiomyopathy, 214 outpatients were prospectively studied from 1980 to 1997 at the Equipe de Cardiopatias Gerais do Instituto do Coração do Hospital das Clínicas da Faculdade de Medicina da Universidade de São Paulo. There were 102 male and 112 female patients, aged $37 \pm 16$ years, ranging from 3 to 76 years. They underwent electrocardiography, 24-h ambulatory electrocardiographic recording, echocardiography and hemodynamic study. There were 22 deaths, 14 (6\%) of them as a direct consequence of the disease, after a mean follow up of $88 \pm 56$ months $(13$ - 299). Eleven of these deaths (78\%) were sudden and unexpected. The cumulative survival rates were $93.8 \%$ in 5 years, $87.7 \%$ in 10 years and $76.4 \%$ in 15 years. The annual mortality rate was 0.4\%. Univariate analysis was performed taking into account 23 clinical variables and 20 variables obtained from laboratory tests. Only family history was shown to be associated with cardiac mortality. In addition, four known adverse factors such as family history, young age, syncope, and functional class were chosen for entering a Cox's multivariate stepwise model. Again, only family history was identified as an independent risk factor for cardiac death. We concluded, based on this selected population from a referral center, that long-term survival of patients with hypertrophic cardiomyopathy is benign and related to family history. 


\section{NTRODUÇÃO}

A Cardiomiopatia Hipertrófica ( $\mathrm{CMH}$ ) é caracterizada por hipertrofia do miocárdio, que determina aumento da espessura das paredes sem dilatação ventricular, na ausência de outra cardiopatia ou doença sistêmica que possa lerar a hipertrofia. A cavidade do ventrículo esquerdo apresenta-se normal ou reduzida, havendo importante disfunção diastólica, enquanto a função sistólica em repouso é normal ou se observa estado hiperdinâmico ${ }^{1-5}$. Entretanto, a literatura mostra que na fase final da doença, aproximadamente $10 \%$ dos pacientes evoluem com dilatação do ventrículo esquerdo e função sistólica diminuída ${ }^{6,7}$. Sua prevalência é estimada em $0,2 \%$ da população geral e em $0,5 \%$ entre os portadores de cardiopatia ${ }^{8}$. Assim, existiriam aproximadamente 320.000 portadores da moléstia em nosso País.

Apesar da descrição de casos isolados por Hallopeau ${ }^{9}$ e por Liouville ${ }^{10}$ (1869), Schmincke ${ }^{11}$ (1907), Davies ${ }^{12}$ (1952) e Brock ${ }^{13}$ (1957), coube a Teare ${ }^{1}$ (1958) descrever a CMH em oito jovens, sete dos quais faleceram de morte súbita. A moléstia foi caracterizada clinicamente por Goodwin et al. ${ }^{2}$ (1960) e por Braunwald et al. ${ }^{3}$ (1964).

A doença é transmitida geneticamente em aproximadamente $50 \%$ dos pacientes ${ }^{14}$. Estudos recentes realizados em famílias de portadores de $\mathrm{CMH}$ mostram que a moléstia pode ser causada por mutação em um dos quatro genes que codificam proteínas contráteis da fibra miocárdica ${ }^{14-16}$. Estes estão localizados: no cromossomo 14 - o locus responsável pela cadeia pesada da $\beta$-miosina cardíaca, observado em 30\%; no cromossomo 1 - o locus responsável pela troponina T, observado em 15\%; no cromossomo 15 - o locus da $\alpha$-tropomiosina, em 3\%. Por último, é descrita também alteração no cromossomo 11, responsável pela ligação da miosina à proteina $\mathrm{C}$ em $15 \%$. Evidências morfológicas da doença podem ser observadas por meio de estudo ecocardiográfico em $25 \%$ dos parentes de primeiro grau desses pacientes ${ }^{17}$.

As alterações constatadas em necrópsias incluem: hipertrofia do ventrículo esquerdo com espessamento maior do septo interventricular em relação à parede livre 
18. cavidade ventricular diminuída ou normal ${ }^{6,7}$; átrio esquerdo dilatado e valva mitral com alterações em $66 \%$ dos $\operatorname{casos}^{19}$, com ou sem espessamento secundário; placa de fibrose no septo interventricular na via de saída do ventrículo esquerdo, no local do choque da cúspide anterior da valva mitral; artérias coronárias intramurais com espessamento da camada média e diminuição da luz em $80 \%$ dos casos ${ }^{20}$; alteração da arquitetura miocárdica por hipertrofia e desarranjo das fibras, assim como das miofibrilas e da matriz extracelular, com aumento do colágeno e destruição das fibras elásticas ${ }^{21,22}$.

Essas modificações anatômicas determinam disfunções que podem se manifestar clinicamente. Assim, as alterações das artérias coronárias são, possivelmente, responsáveis pela isquemia e fibrose que, associadas a áreas de desarranjo celular, podem ser substrato para arritmias ventriculares associadas à morte súbita, a forma mais freqüente de morte, e que ocorre principalmente em pacientes jovens ${ }^{6,7,23,24}$.

A $\mathrm{CMH}$ tem fisiopatologia bem definida. No entanto, apresenta grande diversidade morfológica, funcional e clínica. Assim, a doença costuma ser diagnosticada entre os 30 e 40 anos de idade, embora seja encontrada também em natimortos e octogenários $^{6,7}$. A maioria dos pacientes é assintomática, porém muitas vezes a morte súbita pode ser a primeira manifestação da $\mathrm{CMH}^{7,16,25}$.

A história natural é de difícil caracterização, pois o conhecimento que temos a respeito vem de pacientes sintomáticos, estudados em centros de referência terciários, não correspondendo à realidade da doença. Isto, sem dúvida, tem influenciado a definição do perfil da enfermidade ${ }^{7,25}$.

Até há pouco tempo admitia-se que a moléstia tinha prognóstico reservado, uma vez que importantes trabalhos mostraram mortalidade anual entre $2 \%$ e $4 \%$ em adultos $^{26-31}$ e entre $5 \%$ e $6 \%$ em crianças ${ }^{27}$. Isto foi observado pelos principais centros de referência localizados nos Estados Unidos, Canadá e Grã-Bretanha. Não há publicações na literatura procedentes da América Latina. Este fato levou Maron ${ }^{7}$, um 
dos maiores estudiosos da doença, a pensar que a mesma não ocorre em outras regiões que não as descritas.

Existem apenas duas publicações, uma da Grã Bretanha ${ }^{32}$ e outra da Itália 33, com casuística pequena (39 e 25 pacientes, respectivamente) e curto seguimento (3,1 e 4,4 anos, respectivamente), de pacientes não selecionados, em que não foram observados óbitos. Os autores concluem que a história natural de pacientes portadores da moléstia pode ser benigna. Outros trabalhos, mais recentes, com maior casuística de pacientes não selecionados e com longo seguimento, mostram mortalidade anual entre $0,5 \%$ e $1,5 \%$, com sobrevida semelhante à da população geral adulta ${ }^{34-39}$.

Desde a descrição da $\mathrm{CMH}$, inúmeros pesquisadores têm procurado estratificar o risco de morte súbita, que permanece ainda indefinido. A morte súbita ocorre entre 12 e 35 anos, sendo rara na primeira década de vida e após os 65 anos. Vários autores relatam como fatores de alto risco para morte súbita o antecedente de parada cárdiorespiratória ou de taquicardia ventricular sustentada, genótipo de risco ou história familiar de morte súbita, taquicardia ventricular não sustentada, síncope recorrente, idade menor que 20 anos e classe funcional III-IV (New York Heart Association) ${ }^{7,16,24,27-29}$.

Desde então, chamou-se a atenção para o fato de que a elevada mortalidade seria decorrente da observação de pacientes selecionados em centros de referência e, portanto, com maior grau de comprometimento, enquanto a maioria dos pacientes sequer tem 0 diagnóstico firmado ${ }^{40}$. Isto pode ser corroborado pelo fato de que os maiores centros que estudam a moléstia possuem não mais que uma ou duas centenas de pacientes catalogados.

Estas dúvidas referentes à casuística, local de estudo e sobrevida, despertaram o interesse em se conhecer a história natural desses doentes em nosso meio.

Nosso objetivo foi estudar a sobrevida de pacientes portadores de $\mathrm{CMH}$, assim como observar possíveis fatores determinantes de sobrevida. 


\section{CASUÍ STI CA E MÉTODO}

Foram estudados 214 pacientes com diagnóstico de $\mathrm{CMH}$ matriculados no Ambulatório de Cardiopatias Gerais do Instituto do Coração do Hospital das Clínicas da Faculdade de Medicina da Universidade de São Paulo - Hospital de referência terciária da rede do Estado e também do País.

Todos os pacientes foram acompanhados pelo autor, de forma prospectiva (período mínimo de 12 meses), a partir de 1980.

\section{Seleção de Pacientes}

Os pacientes procuraram o Hospital para avaliação de sintomas, foram encaminhados por outros médicos para avaliação de sopros cardíacos ou eram parentes assintomáticos de portadores da doença.

Todos tiveram diagnóstico clínico realizado no próprio hospital por meio de anamnese, exame físico e confirmado pela ecocardiografia.

Foram excluídos pacientes com hipertensão arterial e com doença cardíaca ou sistêmica que pudesse levar a hipertrofia do miocárdio.

\section{Duração do Estudo}

O estudo teve início em janeiro de 1980 e foi encerrado em março de 1997.

\section{Avaliação Clínica}

Os pacientes foram acompanhados por um período de 13 a 299 meses ( média $=88 \pm 56$ ).

As avaliações clínicas foram realizadas a cada quatro meses, sendo este período abreviado, quando necessário. 
Consideraram-se os seguintes sintomas: presença ou não de dispnéia, palpitações, precordialgia, pré-síncope e síncope. Pela intensidade dos sintomas os pacientes foram classificados funcionalmente de acordo com os critérios da New York Heart Association (CF - NYHA).

Foram considerados os seguintes sinais: presença ou não de sopro da via de saída do ventrículo esquerdo e/ou de insuficiência mitral, pulso e pressão arterial sistólica e diastólica, medida na posição supina.

Os pacientes foram considerados como tendo a forma familiar quando a doença estava presente em algum parente de primeiro grau com diagnóstico clínico e ecocardiográfico.

\section{Avaliação Eletrocardiográfica}

O eletrocardiograma de repouso foi realizado em todos os pacientes nas 12 derivações clássicas, em aparelho da marca Hewlett-Packard, de um canal de registro até 1980 e, a partir daí, em aparelho de três canais, sendo que a partir de 1990 no modelo 4745-A (page writer), todos de inscrição direta, velocidade do papel de 25 milímetros por segundo e calibração de um centímetro.

A análise do eletrocardiograma foi feita por dois cardiologistas, separadamente, obedecendo critérios internacionalmente aceitos ${ }^{41}$.

Eletrocardiografia dinâmica de 24 horas foi realizada em 172 pacientes em duas derivações precordiais (CM5 e CM1), a partir de 1979, em aparelho Del Mar Avionics (modelo 445A), com tempo de gravação de 24 horas. Os registros foram analisados por computador Del Mar Avionics (modelo 660B) e software 17059 para quantificação, tabulação e impressão. A partir de 1990 os registros foram realizados em aparelho Marquette 8000 e a análise realizada em processador Marquette Laser SXP e software versão 5.8 .

As variáveis analisadas foram: freqüência cardíaca média, número de extrassístoles supraventriculares por hora, número e tipo de extrassístoles ventriculares 
por hora e presença ou não de taquicardia ventricular não sustentada (três ou mais batimentos consecutivos).

\section{Avaliação da Ecocardiografia com Doppler}

Os exames ecocardiográficos foram realizados em equipamentos comercialmente disponíveis na Institutição, utilizando-se transdutores setoriais, mecânicos ou eletrônicos, com freqüências de 2,5 a 3,5 MHz, sendo os registros gravados em papel termo-sensível ou em videotape com utilização dos modos $M$ (unidimensional), bidimensional e da técnica de Doppler.

As imagens da ecocardiografia bidimensional obtidas do ventrículo esquerdo, através de incidências padronizadas, foram utilizadas para avaliar, em tempo real, o padrão de contração regional e global e da estrutura ventricular, e também para ajudar na obtenção do modo M. As medidas das dimensões internas do ventrículo esquerdo foram obtidas através do modo $M$, no final da sístole e final da diástole, com auxílio de registro eletrocardiográfico simultâneo, sendo realizadas conforme recomendações da Sociedade Americana de Ecocardiografia, assim como as medidas das espessuras do septo interventricular e da parede livre no final da diástole. A avaliação ecocardiográfica também possibilitou verificar a presença do movimento anterior sistólico da valva mitral e do fechamento mesossistólico da valva aórtica. A técnica de Doppler foi utilizada na detecção e quantificação de regurgitações valvares, e também na detecção, quantificação e cálculos para a estimativa de gradientes de pressão intraventricular ${ }^{42-45}$.

As medidas dos diâmetros internos do ventrículo esquerdo, obtidas no final da sístole e da diástole, permitiram os cálculos dos volumes diastólico e sistólico finais, e do volume sistólico (em mililitros), conforme metodologia já estabelecida, assim como os cálculos de função ventricular, através da fração de encurtamento e da fração de ejeção (em porcentagem). As variáveis analisadas, portanto, foram: espessuras do septo interventricular e da parede livre do ventrículo esquerdo no final da diástole (em milímetros), dimensão do átrio esquerdo (em milímetros), diâmetro interno do 
ventrículo esquerdo no final da diástole (em milímetros), fração de ejeção (em porcentagem) ${ }^{46-48}$, presença de movimento anterior sistólico da valva mitral e de fechamento mesossistólico da valva aórtica, presença de regurgitação mitral e gradiente de pressão na via de saída do ventrículo esquerdo (em milímetros de mercúrio).

Foram utilizados critérios de normalidade já estabelecidos para as medidas das dimensões ventriculares ${ }^{49}$. Em relação à função sistólica global do ventrículo esquerdo foram considerados como normais valores para a fração de encurtamento acima de $30 \%$ e para a fração de ejeção acima de 0,65 . Com respeito à relação septo/parede foram considerados anormais valores maiores que 1,3. A presença do movimento anterior sistólico da valva mitral e do fechamento mesossistólico da valva áortica, independentemente da deteç̧ão de gradiente de pressão intraventricular na via de saída do ventrículo esquerdo, foi utilizada como critério para caracterizar os pacientes com forma obstrutiva em repouso.

\section{Avaliação Hemodinâmica}

Foi realizada em 50 pacientes, utilizando-se técnicas padronizadas para medida da pressão sistólica na ponta do ventrículo esquerdo e na via de saída do mesmo, considerando-se significativo o gradiente de pressão intraventricular maior que 30 milímetros de mercúrio em repouso.

\section{Tipo de Tratamento e Resposta}

Os pacientes foram submetidos a tratamento clínico com drogas, cirurgia clássica de cardiomiectomia transvalvar aórtica ou implante de marcapasso.

\section{Tratamento Clínico}

As drogas utilizadas foram: bloqueadores de receptores $\beta$-adre-nérgicos (propranolol, atenolol, nadolol, metoprolol ou sotalol); bloqueadores de canais de cálcio (verapamil, nifedipina), disopiramida e amiodarona. Esta última foi administrada 
em baixa dose aos pacientes com taquicardia ventricular não sustentada à eletrocardiografia dinâmica, e naqueles com fibrilação atrial paroxística.

Os pacientes assintomáticos não receberam medicação. Pacientes com sintomas e sinais conseqüentes à disfunçâo sistólica e à dilatação ventricular foram medicados com digital, diuréticos e drogas inibidoras da enzima conversora da angiotensina. Pacientes com fibrilação atrial crônica foram medicados com warfarin.

Resposta ao Tratamento

Por ocasião da última consulta, os pacientes foram avaliados com relação ao seu estado funcional: assintomáticos ou melhorados, estáveis ou sem melhora e com piora.

\section{Evolução}

A evolução foi realizada em nossa Instituição. No caso de morte fora da Instituição, as informações foram obtidas a partir de familiares ou de médicos que haviam acompanhado o paciente nesse evento. Foi definido o tipo de óbito - se por causa cardíaca (morte súbita, insuficiência cardíaca congestiva ou endocardite infecciosa), não cardíaca, ou óbito de causa desconhecida. Morte súbita foi definida quando ocorreu até uma hora após o início dos sintomas.

\section{Análise Estatística}

0 grupo de pacientes que faleceram foi comparado com o grupo de pacientes que sobreviveram em relação às variáveis qualitativas, através dos testes de Qui-quadrado de Pearson e exato de Fisher. As variáveis quantitativas foram analisadas através do teste $t$ de Student ${ }^{50}$.

As variáveis estudadas foram:

\section{- clínicas}

- idade, sexo, forma familiar, forma obstrutiva 
- sintomas: dispnéia, precordialgia, palpitações, síncope, pré-síncope e classe funcional

- sinais: pulso, pressão arterial sistólica, pressão arterial diastólica, sopro sistólico em borda esternal esquerda e sopro sistólico em área mitral

- eletrocardiograma: fibrilação atrial, sobrecarga atrial esquerda e sobrecarga ventricular esquerda

- eletrocardiografia dinâmica: freqüência cardíaca média, extrassístoles supraventriculares, extrassístoles ventriculares e taquicardia ventricular não sustentada

- ecocardiografia com Doppler: espessura diastólica do septo interventricular, espessura diastólica da parede livre do ventrículo esquerdo, relação septo/parede, tamanho do átrio esquerdo, tamanho do ventrículo esquerdo, fração de ejeção do ventrículo esquerdo, movimento anterior sistólico da valva mitral, fechamento mesossistólico da valva aórtica, insuficiência mitral e gradiente da via de saída do ventrículo esquerdo

- estudo hemodinâmico: pressão média da ponta do ventrículo esquerdo, pressão média da via de saída do ventrículo esquerdo e gradiente médio da via de saída do ventrículo esquerdo

- tratamento: clínico, cirúrgico ou marcapasso

- resposta ao tratamento: assintomático ou melhorado, estável ou sem melhora e piora

- medicação: propranolol, verapamil ou amiodarona.

0 método de Kaplan-Meier e o teste de Log-Rank foram empregados no estudo da sobrevida de pacientes com relação às variáveis: sexo, idade, forma familiar, palpitação, síncope, classe funcional, tratamento, taquicardia ventricular não sustentada, espessura diastólica do septo interventricular, relação septo/parede, movimento anterior sistólico da valva mitral, fechamento mesossistólico da valva 
aórtica e gradiente da via de saída do ventrículo esquerdo pela ecocardiografia com Doppler e pelo estudo hemodinâmico ${ }^{51}$.

A taxa de mortalidade total foi calculada da seguinte forma: número de pacientes que foram a óbito dividido pelo número total de pacientes estudados. Assim sendo, a taxa de mortalidade anual obteve-se dividindo a taxa total pelo tempo de acompanhamento.

O modelo de regressão de Cox foi utilizado para estudo multivariado de sobrevida em função das variáveis: classe funcional, idade, forma obstrutiva e forma familiar. O método Stepwise foi aplicado com a intenção de selecionar as variáveis associadas

\section{RESULTADOS}

\section{Caracterização Clínica}

Dos 214 pacientes estudados, 112 eram do sexo feminino com idades entre 3 e 76 anos (média 37士16), por ocasião da primeira consulta (Gráfico 1).

Em 28 (13,08\%) pacientes constatou-se forma familiar e em $114(53,27 \%)$ forma obstrutiva.

Vinte pacientes $(9,34 \%)$ eram assintomáticos, 145 (67,76\%) referiam dispnéia, 82 (38,32\%) precordialgia, 64 (29,91\%) palpitações, 33 (15,42\%) síncope e $10(4,67 \%)$ pré-síncope. Sessenta e seis pacientes $(30,84 \%)$ encontravam-se na classe funcional I, $127(59,34 \%)$ na II, $20(9,34 \%)$ na III e um $(0,47 \%)$ na IV.

A freqüência cardíaca de todos os pacientes e as pressões arteriais sistólica e diastólica encontravam-se dentro dos limites da normalidade. 
Em 164 (76,63\%) pacientes observou-se sopro sistólico do tipo ejeção em borda esternal esquerda baixa e em 112 (52,34\%) sopro sistólico de regurgitação em área mitral (Tabela 1).

Duzentos e três (95\%) pacientes estavam recebendo medicação: 115 em uso de drogas bloqueadoras de receptores $\beta$-adrenérgicos, 76 drogas bloqueadoras de canais de cálcio, 38 amiodarona e três disopiramida (Tabela 2).

Não foi observada diferença estatisticamente significante entre o grupo de pacientes vivos e o de pacientes que evoluíram para óbito com relação às variáveis idade (maior ou menor que 20 anos), sexo, forma familiar, forma obstrutiva, dispnéia, precordialgia, síncope, pré-síncope, freqüência cardíaca, pressão arterial sistólica, diastólica, presença de sopro da via de saída do ventrículo esquerdo e insuficiência mitral. Apenas a variável palpitação foi associada ao óbito, de modo que a proporção de óbitos foi significantemente maior nestes pacientes (Tabela 3).

A análise comparativa entre as categorias idade $(p=0,569)$, sexo $(p=0,293)$, palpitação $(p=0,084)$, síncope $(p=0,651)$ e forma familiar $(p=0,064)$ (teste de Log-Rank) mostrou que apenas esta última foi fator de risco (Gráfico 2).

A análise multivariada de Cox para as variáveis idade, classe funcional I-II, III-IV, forma familiar ou não, forma obstrutiva ou não, mostrou que apenas a forma familiar foi fator de risco independente de óbito, confirmado pelo modelo de Cox com Stepwise para a seleção de variáveis $(p=0,074)$. Os pacientes com forma familiar apresentam risco de óbito 2,5 vezes maior do que aqueles com a forma não familiar.

\section{Eletrocardiografia}

Dos 214 pacientes, $26(12,15 \%)$ apresentavam eletrocardiograma normal. Em 179 (83,64\%) observou-se sobrecarga ventricular esquerda, em 36 (16,82\%) sobrecarga atrial esquerda, em $18(8,41 \%)$ ritmo de fibrilação atrial, em sete $(3,27 \%)$ síndrome de Wolff-Parkinson-White, em três $(1,40 \%)$ bloqueio de ramo esquerdo do 
feixe de His, em três $(1,40 \%)$ área eletricamente inativa e em dois $(0,93 \%)$ sobrecarga ventricular direita (Tabela 4).

Não foi observada diferença estatisticamente significante entre o grupo de pacientes vivos e o de pacientes que evoluíram para óbito com relação às variáveis fibrilação atrial, sobrecarga atrial e sobrecarga ventricular esquerda (Tabela 5).

\section{Eletrocardiografia Dinâmica}

Dos 172 pacientes que realizaram o exame, em 153 (88,95\%) observaramse arritmias, sendo em 98 (56,97\%) supraventriculares e em 141 (81,97\%) ventriculares. As arritmias supraventriculares foram: extrassístoles atriais em 92 $(53,49 \%)$, mais do que 30 por hora em quatro pacientes $(2,32 \%)$, taquicardia atrial paroxística em um paciente $(0,58 \%)$ e fibrilação atrial em outro paciente $(0,58 \%)$.

As arritmias ventriculares foram: extrassístoles ventriculares, mais do que dez por hora em 34 (19,76\%) pacientes, polimórficas em 35 (20,35\%), pareadas em $45(26,16 \%)$ e taquicardia ventricular não sustentada (monomórfica, com menos de dez batimentos e freqüência <150) em $33(19,18 \%)$ (Tabela 6).

Não foi observada diferença estatisticamente significante entre o grupo de pacientes vivos e o grupo de pacientes que evoluíram para óbito em relação às variáveis freqüência cardíaca média, extrassístoles ventriculares e presença ou não de taquicardia ventricular não sustentada. Os grupos foram diferentes com relação às extrassístoles supraventriculares, de modo que a média destas foi significantemente menor nos pacientes que evoluíram para óbito (Tabela 7).

A análise de sobrevida através do teste de Log-Rank, para a variável taquicardia ventricular não sustentada, não mostrou ser esta fator de risco de óbito $(p=0,512)$. 


\section{Estudo da Ecocardiografia com Doppler}

Os valores obtidos foram: espessura do septo interventricular $21 \pm 5 \mathrm{~mm}$ (13-39), parede livre do ventrículo esquerdo $11 \pm 3 \mathrm{~mm}$; relação septo/parede

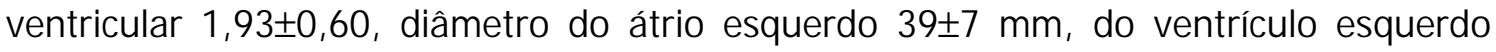
em diástole $42 \pm 6 \mathrm{~mm}$; gradiente da via de saída do ventrículo esquerdo $47 \pm 30 \mathrm{mmHg}$

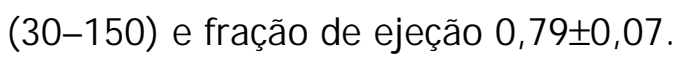

Movimento anterior sistólico da valva mitral foi observado em 146 (68,22\%) pacientes, fechamento mesossistólico da valva aórtica em 120 (56,07\%) e insuficiência da valva mitral em $83(38,78 \%)$ (Tabela 8$)$.

Não foi observada diferença estatisticamente significante entre o grupo de pacientes vivos e o grupo de pacientes que evoluíram para óbito para as variáveis ecocardiográficas: espessura diastólica do septo interventricular, espessura diastólica da parede livre do ventrículo esquerdo, relação septo/parede, tamanho do átrio esquerdo, tamanho do ventrículo esquerdo em diástole, fração de ejeção do ventrículo esquerdo, gradiente da via de saída do ventrículo esquerdo e insuficiência da valva mitral. A porcentagem de óbitos foi significantemente maior no grupo de pacientes que apresentavam movimento anterior sistólico da valva mitral e fechamento mesossistólico da valva aórtica (Tabela 9).

A análise de sobrevida em função das variáveis - espessura do septo interventricular (>20 mm e <20 mm), movimento anterior sistólico da valva mitral e fechamento mesossistólico da valva aórtica, através do teste de Log-Rank, não mostrou serem estas fatores de risco de óbito $(p=0,341,0,177$ e 0,126, respectivamente).

\section{Estudo Hemodinâmico}

Foi observada pressão média na ponta do ventrículo esquerdo de $168 \pm 44$ mmHg e de $125 \pm 29$ na via de saída. O gradiente médio de pressão da via de saída do 
ventrículo esquerdo, em repouso, foi de $66 \pm 29 \mathrm{mmHg}$, variando de 30 a 150 (Tabela 10).

Não foi observada diferença estatisticamente significante para essas variáveis entre 0 grupo de pacientes vivos e o grupo de pacientes que evoluíram para óbito (Tabela 11).

\section{Tratamento e Resposta}

Ao final do estudo 11/192 (6\%) pacientes não usavam medicação e 181/192 (94\%) ainda recebiam medicação. Vinte pacientes (9\%) foram submetidos a tratamento cirúrgico com realização de cardiomiectomia transvalvar aórtica e 13 (6\%) receberam marcapasso definitivo (com modo de estimulação DDD em 9 casos e VVI em quatro), em ambas situações por refratariedade ao tratamento clínico. Todos os pacientes nos quais foi implantado marcapasso e 18 dos submetidos ao tratamento cirúrgico continuaram em uso de medicação.

A resposta global ao tratamento foi: 168/214 (78\%) pacientes tornaram-se assintomáticos, 41/214 (19\%) continuaram estáveis e apenas 5/214 (2\%) pioraram. Cento e sessenta e nove (79\%) pacientes estavam em CF I, 34 (16\%) em CF II, oito (4\%) em CF III e três (1\%) em CF IV.

Quatro pacientes (2\%) evoluíram com sintomas de insuficiência cardíaca, disfunção sistólica, dilatação do ventrículo esquerdo e diminuição da espessura da parede do ventrículo esquerdo. Um deles veio a falecer enquanto aguardava transplante cardíaco.

Não foi observada diferença estatisticamente significante entre o grupo de pacientes vivos e o de pacientes que evoluíram para óbito com relação ao tipo de tratamento, se clínico, cirúrgico ou implante de marcapasso, ou em relação ao tipo de droga que estavam recebendo. No entanto, a porcentagem de óbitos foi maior no grupo de pacientes que não responderam ao tratamento (Tabela 12). 


\section{Evolução}

Ao final do seguimento havia $192(89,72 \%)$ pacientes vivos e $22(10,28 \%)$ falecidos. A idade média por ocasião do óbito foi de $34 \pm 16$ anos, sendo 13 pacientes do sexo masculino, com tempo médio de seguimento de $77 \pm 55$ meses (Tabela 13).

Do total de óbitos $14(6,54 \%)$ foram relacionados à $\mathrm{CMH}$. Onze $(5,14 \%)$ faleceram subitamente, dois de insuficiência cardíaca e um de endocardite infecciosa. Ocorreram 12 óbitos no grupo clínico e dois no grupo submetido a tratamento cirúrgico de cardiomiectomia transvalvar aórtica. Não ocorreu óbito no grupo de pacientes que receberam marcapasso.

Os oito óbitos $(3,74 \%)$ não relacionados à doença foram: três mortes acidentais (dois atropelamentos e um acidente automobilístico), um decorrente de acidente vascular cerebral, um de pneumonia, um de cirrose hepática, um de neoplasia do aparelho digestivo e um de insuficiência renal crônica (Tabela 14).

A estimativa da probabilidade cumulativa de sobrevida (método de KaplanMeier) mostrou, para cinco anos 93,8\%, para dez anos $87,7 \%$ e para 15 anos $76,4 \%$. A taxa de mortalidade anual para todo o grupo de pacientes foi de $0,4 \%$ (Gráfico 3 ).

Do total de 214 pacientes, 93 (43\%) foram acompanhados durante cinco anos, $70(33 \%)$ durante dez anos, $37(17 \%)$ durante 15 anos, $13(6 \%)$ durante 20 anos e apenas um $(0,4 \%)$ pelo período de 25 anos

\section{DISCUSSÃO}

\section{O Risco da Avaliação de Pacientes Selecionados}

A CMH é uma doença complexa que sempre despertou o interesse dos pesquisadores e, apesar de quatro décadas já se terem passado desde sua descrição, 
mesmo com o grande desenvolvimento dos métodos diagnósticos nesse período, ainda existem muitas dúvidas em relação à mesma.

Após a descrição, no final da década de 50, da caracterização clínica, que permitiu o diagnóstico, e da abordagem terapêutica no início dos anos 60, o interesse dos pesquisadores voltou-se para o estudo da história natural da doença e dos fatores prognósticos.

0 primeiro trabalho foi publicado em 1968 por Frank; Braunwald ${ }^{53}$, que seguiram 126 pacientes com diagnóstico de $\mathrm{CMH}$, confirmado por estudo hemodinâmico e angiocardiográfico, por um período de até 12 anos em centro de referência (The Cardiology Branch, National Institute of Health - EUA). Registraram 14 óbitos, dez de origem cardíaca, sendo que destes, seis faleceram de morte súbita. Notaram ainda que nos pacientes em CF I ocorreu 1/42 óbito (2\%); naqueles em CF II, 5/40 óbitos (12\%) e na CF III-IV, 4/16 óbitos (25\%). A idade média dos pacientes era de 29 anos. Não compararam o grupo de pacientes vivos com 0 de pacientes que faleceram para determinar fatores de risco.

Em 1974 foi publicado um estudo cooperativo multicêntrico retrospectivo por Shah et al. ${ }^{26}$ realizado em centros de referência dos Estados Unidos da América, Canadá e Reino Unido, com 190 pacientes e seguimento médio de 5,2 anos. 0 diagnóstico foi clínico, hemodinâmico e angiocardiográfico, sendo que todos os pacientes apresentavam gradiente da via de saída do ventrículo esquerdo em repouso ou provocado. Observaram 49 óbitos (26\%), dos quais 26 foram por morte súbita. A mortalidade anual foi de 3,4 \% e não foram observados fatores de risco de morte súbita entre cinco variáveis clínicas analisadas. Concluíram que a doença não é benigna.

McKenna et al. ${ }^{27}$ (1981) publicaram estudo retrospectivo de 254 pacientes com idades variando entre 1 e 66 anos e seguimento médio de 6 anos realizado em centro de referência (Royal Postgraduate Medical School, Hammersmith Hospital Londres). Observaram 58 óbitos (23\%), dos quais 32 ocorreram por morte súbita, seis 
por insuficiência cardíaca congestiva e dois por endocardite infecciosa. A mortalidade anual para crianças foi de $5,9 \%$ e para adultos de $2,5 \%$. Concluíram que são fatores de risco: a idade por ocasião do diagnóstico em menores de 14 anos, forma familiar com história da doença e de morte súbita, presença de síncope e CF III e IV. Este foi o trabalho que influenciou toda a década de 80 , firmando o conceito de malignidade da doença, principalmente quando diagnosticada em crianças, e que permanece até hoje como a maior casuística já publicada. 0 grupo de pacientes com mais de 60 anos é de apenas sete casos. Foi o primeiro trabalho a utilizar o ecocardiograma modo $\mathrm{M}$ para $\mathrm{o}$ diagnóstico, em alguns pacientes, método este introduzido a partir de 1970, fazendo também referência à eletrocardiografia dinâmica, introduzida em 1979. Nesta época começamos a estudar a doença no Instituto do Coração do Hospital das Clínicas da Faculdade de Medicina da Universidade de São Paulo.

Shapiro; Zezulka ${ }^{32}$ (1983) publicaram o primeiro trabalho com 39 pacientes não selecionados (Dudley Road Hospital, Birmingham), com seguimento médio de 3,1 anos, não tendo sido observado nenhum óbito nesse período. Concluíram que a mortalidade não é diferente da que ocorre na população geral e que a doença não tem prognóstico ruim. Apesar da importância dos resultados, este trabalho não modificou a forma como era vista a $\mathrm{CMH}$, quiçá pela casuística pequena e o curto período de seguimento.

Koga et al. ${ }^{28}$ (1984) mostraram sua experiência com 136 pacientes em centro de referência no Japão, com seguimento médio de 5,1 anos, na qual observaram 21 óbitos (15\%), sendo 18 de causa cardíaca, dos quais 14 faleceram subitamente. A mortalidade anual foi de $3,7 \%$ e a probabilidade de sobrevida em cinco anos de $87 \%$ e em dez anos de $68 \%$. Concluíram que a morte súbita foi a forma como os pacientes faleceram e que apenas a idade $<20$ anos foi fator de mau prognóstico, uma vez que estes pacientes tiveram mortalidade anual por morte súbita de $7,9 \%$, sendo o risco relativo oito vezes maior que o dos pacientes mais idosos.

Em 1989, Spirito et al. ${ }^{33}$ publicaram sua experiência na Itália com 25 pacientes não selecionados e seguimento médio de 4,4 anos, sem registro de óbito, 
após terem excluído um óbito de origem não cardíaca. Apesar desta casuística ser pequena, é semelhante à descrita na literatura em relação à idade, sexo, extensão da hipertrofia, alterações da função diastólica, obstrução da via de saída do ventrículo esquerdo e arritmias ventriculares. Concluíram que a história natural da $\mathrm{CMH}$ pode ser mais benigna que a relatada em centros de referência. Chamaram a atenção, após levantamento de 78 artigos publicados em quatro das revistas mais importantes de Cardiologia (American Journal of Cardiology, Circulation, The Journal of American College of Cardiology e The British Heart Journah), no período de 1983 a 1987 com 3404 pacientes com $\mathrm{CMH}$, que $50 \%$ dos trabalhos com $70 \%$ dos pacientes procedem de dois centros de referência: o National Institutes of Health de Bethesda, Md., EUA e o Royal Postgraduate School, Londres, RU. Estes desenharam o perfil da doença e nos propuseram as linhas de conduta.

Nos anos 90 foram publicados os trabalhos de Romeo et al. ${ }^{29}$, com mortalidade anual de $3,4 \%$; de Fay et al. ${ }^{30}$, em pacientes septuagenários, que observaram mortalidade anual de 3\% e probabilidade de sobrevida de $95 \%$ em um ano e $76 \%$ em cinco anos; de Seiler et al. ${ }^{31}$, que observaram mortalidade anual de 3,6\% e probabilidade de sobrevida de $78 \%$ em cinco anos e $67 \%$ em dez anos; de Azzano et al. ${ }^{54}$, que observaram mortalidade anual de $3,2 \%$ em pacientes jovens com idade $<20$ anos e probabilidade de sobrevida de $90 \%$ em cinco anos e $85 \%$ em dez anos. Todos eles avaliaram pacientes selecionados em centros de referência.

No início desta década começaram a ser publicados trabalhos com pacientes não selecionados e com casuísticas consideráveis, com longo seguimento, que vêm mostrar que a doença é benigna e a sobrevida, se não é igual, fica muito próxima daquela observada na população geral.

0 primeiro estudo foi publicado por Kofflard et al. ${ }^{34}$ (1993), com mortalidade anual de 1\%. Em 1995, Cannan et al. ${ }^{35}$, baseados em uma população do Condado de Olmsted, Minnesota, EUA, observaram mortalidade anual de 0,7\%, com probabilidade de sobrevida de $95 \%$ em um ano e $92 \%$ em cinco anos. No mesmo ano Cecchi et al. ${ }^{36}$ publicaram estudo realizado na Toscana, Itália, que mostrou 
mortalidade anual de 0,6\% e sobrevida cumulativa de $97 \%$ para cinco anos, $95 \%$ para dez anos e $92 \%$ para 15 anos.

Recentemente, Maron et al. ${ }^{37}$, estudando 227 pacientes não selecionados em Minnesota, EUA, não observaram diferença de sobrevida entre os maiores de 20 anos, após ajuste de sexo, idade e raça, quando comparados com tabelas de sobrevida para população geral dos EUA. Porém, idade menor que 20 anos foi fator de risco de óbito.

Como fica evidente, pelo exposto, a avaliação de pacientes selecionados em centros de referência está sujeita a críticas. As informações dos variados grupos deve ser analisada cuidadosamente e todos os centros de estudo devem ter a sua própria experiência.

\section{Sobrevida}

Nossa casuística, de 214 pacientes, é a quarta em número desde a descrição da doença. Trata-se de população jovem com idade média de 37 anos, próxima à da maioria dos trabalhos 27-29,31,34,36. Há discreto predomínio do sexo feminino, diferentemente da literatura ${ }^{26-29,31-37}$, em que predominam pacientes do sexo masculino, à exceção da casuística de Fay et al. ${ }^{30}$, constituída de pacientes idosos, em que $61 \%$ eram mulheres. Observamos incidência de $13 \%$ de formas familiares, bem menor que a observada por outros autores, que oscila entre 20 e 53\% 26,27,29,32,34,36,54. Isto, sem dúvida, foi devido aos critérios por nós adotados na seleção dos pacientes (Tabela 15).

As formas obstrutivas foram observadas em $53 \%$ de nossos pacientes, maior do que o observado por outros autores ${ }^{27,30,34-36,54}$, entre 20 e $52 \%$. Com relação à expressão da doença, ao grau de hipertrofia miocárdica e à prevalência de taquicardia ventricular não sustentada, os achados são semelhantes aos previamente descritos na literatura. Por outro lado, no que diz respeito ao quadro clínico, nossa casuística, de pacientes também selecionados em centro de referência, difere consideravelmente da literatura, uma vez que apenas $10 \%$ encontravam-se em CF III- 
IV, sendo que a maioria dos autores observou $20-65 \%$ dos pacientes nessa condição 26,27,29,32,53,54. Nossos resultados estão mais de acordo com aqueles observados em pacientes não selecionados ${ }^{33-36}$ (Tabela 15). Com o tratamento, clínico ou cirúrgico, obtivemos resposta satisfatória em $97 \%$ dos pacientes e, ao final do estudo, apenas $4 \%$ encontravam-se em CF III-IV.

Nossos resultados mostram, pela primeira vez, que a sobrevida da $\mathrm{CMH}$ em uma população de pacientes selecionados com seguimento de longa duração em centro de referência é, provavelmente, semelhante à da população geral, com mortalidade anual de $0,4 \%$ ao ano, bem menor que a referida nos trabalhos clássicos da literatura e que oscila entre 3-4\% para adultos ${ }^{26-31}$ e $6 \%$ para crianças ${ }^{27}$, sendo semelhante àquela descrita mais recentemente em levantamentos populacionais de pacientes não selecionados e que oscila entre $0-1 \%{ }^{32-37}$ (Tabela 15). Lamentavelmente, não existem em nosso País, tabelas de sobrevida para a população geral ajustada à idade, sexo e raça.

Entretanto, a probabilidade de sobrevida de nossos pacientes, calculada em $93,8 \%$ para cinco anos e em $87,7 \%$ para dez anos, é maior que a observada por Koga et al. ${ }^{28}$, de $87 \%$ e $68 \%$ e que a do grupo clínico de Seiler et al. ${ }^{31}$, de $78 \%$ e $67 \%$ e muito próxima da observada por Fay et al. ${ }^{30}$, de $95 \%$ e $76 \%$, (grupo controle de $97 \%$ e $82 \%$ ), do grupo cirúrgico de Seiler et al. ${ }^{31}$, de $96 \%$ e $84 \%$, de Azzano et al. ${ }^{54}$, de $90 \%$ e $85 \%$, de Cannan et al. ${ }^{35}$, de $92 \%$ para cinco anos (grupo controle de $87 \%$ ), de Cecchi et al. ${ }^{36}$, de $97 \%$ e $95 \%$ e de Yoshida et al. ${ }^{55}$, de $98 \%$ e $89 \%$. A probabilidade de sobrevida dos pacientes estudados para 15 anos é de $76,4 \%$, menor do que a observada por Cecchi et al. ${ }^{36}$, de $92 \%$. Isto pode ser explicado por serem, possivelmente, casuísticas constituídas por pacientes geneticamente diferentes.

\section{Fatores Prognósticos}

Desde que Teare ${ }^{1}$ (1958) descreveu a CMH em oito adultos jovens (idade média 27 anos), sete dos quais tinham falecido de morte súbita, muitos pesquisadores têm procurado estratificar o risco de morte súbita, que é a forma mais freqüente de morte nesta moléstia ${ }^{7,24,56}$. 
Em nosso grupo de pacientes com CMH observamos que a morte súbita foi a forma mais freqüente de óbito e ocorreu em 11/14 (78\%) dos relacionados à doença, o que está de acordo com o observado por outros autores e que oscilou entre 50 e $82 \%$ 26-28,34,35,37,53,54. Estes dados diferem dos de Cecchi et al. ${ }^{36}$, que relataram 2/13 (15\%) óbitos por morte súbita e que também, contrariamente à literatura, observaram que a maioria (11/13) faleceu com sintomas de insuficiência cardíaca congestiva; destes, apenas seis (3\%) tinham diminuição da hipertrofia e fração de ejeção reduzida. Nós observamos que apenas $2 \%$ dos pacientes evoluíram para a fase final da doença com dilatação do ventrículo esquerdo, diminuição da espessura das paredes e da fração de ejeção, o que está de acordo com o observado por este último autor, porém diferente da literatura, que mostra esse tipo de evolução para fase final em $10 \%$ dos pacientes ${ }^{7,16,25}$.

As outras formas de óbito não diferem das observadas pela maioria dos autores, inclusive as mortes por outras causas, que não de origem cardíaca e que ocorrem naturalmente em qualquer população seguida por longo período de tempo ${ }^{27-}$ 34,53

Em revisão recente, Maron ${ }^{7}$ relatou como fatores de alto risco para morte súbita o antecedente de parada cardio-respiratória ou de taquicardia ventricular sustentada, genótipo de risco ou história familiar de múltiplas mortes súbitas, taquicardia ventricular não sustentada, síncope recorrente e grandes hipertrofias ventriculares com espessura maior que 35 milímetros.

Nós analisamos 23 variáveis clínicas e observamos que apenas a variável forma familiar foi fator independente de risco de óbito. Assim, pacientes sem forma familiar apresentam probabilidade de sobrevida de 93,9\% para cinco anos, de 90,0\% para dez anos e de $80,9 \%$ para 15 anos, enquanto na presença de forma familiar a probabilidade de sobrevida é, respectivamente, de $92,8 \%, 65,3 \%$ e de $32,6 \%$.

Os pacientes com forma familiar têm risco de óbito 2,5 vezes maior do que aqueles com forma não familiar. Isto está de acordo com a literatura e o primeiro a chamar a atenção para esta variável como fator de risco foi McKenna et al. ${ }^{27}$. Mais recentemente, com 0 descobrimento de defeitos em gens que determinam a 
codificação das proteínas contráteis do sarcômero, foi enfatizada a importância das formas familiares e, para alguns tipos de alteração cromossômica têm sido descritas formas com prognóstico ruim ${ }^{14,15}$.

A análise de 20 variáveis obtidas pela eletrocardiografia, eletrocardiografia dinâmica, ecocardiografia com Doppler e estudo hemodinâmico, para avaliação da morfologia e da função cardíaca, não conseguiu determinar outros fatores de risco de mau prognóstico. Isto, possivelmente, é decorrente do pequeno número de óbitos de nossa casuística. Por outro lado, os pacientes com "alto risco" pelos critérios relatados por vários autores, constituem minoria e estariam no ápice da pirâmide da estratificação proposta por Maron ${ }^{7}$.

Acreditamos ainda que a idade menor que 20 anos é fator importante de risco de óbito, uma vez que continua sendo significativa em estudos de pacientes não selecionados ${ }^{37}$. Em nossa casuística não foi fator de risco, possivelmente pelo pequeno número de pacientes com menos de 20 anos. Porém, a idade média dos pacientes que faleceram foi menor que a dos sobreviventes (34 e 37 anos, respectivamente).

Sem dúvida, a evolução clínica e a história natural resultam de complexa interação entre a hipertrofia ventricular, o remodelamento cardíaco e alterações funcionais como a disfunção diastólica, isquemia miocárdica, obstrução da via de saída do ventrículo esquerdo e arritmias. Essas alterações morfológicas e funcionais variam de paciente para paciente, talvez por influência genética, fazendo com que tanto a evolução clínica como a história natural sejam variáveis. Além disso, a população conhecida é constituída pelos pacientes, enquanto a população maior, assintomática, é desconhecida por nós, uma vez que esta sequer sabe ser portadora da doença.

Porém, nossos resultados mostram que tanto a evolução clínica como a história natural, a longo prazo, são melhores do que a que nos foi mostrada durante três décadas, a partir de pacientes selecionados em centros de referência. 


\section{CONCLUSÕES}

1. Em nosso meio houve maior incidência de $\mathrm{CMH}$ em adultos jovens, com discreto predomínio do sexo feminino. A maioria dos pacientes, apesar de selecionada em centro de referência, era pouco sintomática ( $31 \%$ em CF I e $59 \%$ em CF II), tendo apresentado resposta satisfatória ao tratamento clínico e/ou cirúrgico (80\% em CF I e $16 \%$ em CF II). Apenas quatro pacientes (2\%) evoluíram com dilatação e diminuição da fração de ejeção do ventrículo esquerdo.

2. A evolução clínica do grupo foi excelente, pois ao final do seguimento havia 192 sobreviventes e 22 óbitos; destes, apenas $14(6 \%)$ relacionados à doença, sendo que morte súbita ocorreu em 78\% (principal causa de morte).

3. A probabilidade cumulativa de sobrevida para cinco anos foi de $93,8 \%$, para dez anos $87,7 \%$ e para 15 anos de $76,4 \%$.

4. A taxa de mortalidade anual para todo o grupo foi de $0,4 \%$.

5. A forma familiar foi considerada fator independente de risco de óbito.

6. Como conclusão final, com base em população selecionada em centro de referência, podemos afirmar que, a longo prazo, a sobrevida de pacientes portadores de cardiomiopatia hipertrófica é benigna. 
TABELA 1. CARACTERIZAÇÃO CLÍNICA DE 214 PACIENTES PORTADORES DE CARDIOMIOPATIA HIPERTRÓFICA POR OCASIÃO DO DIAGNÓSTICO

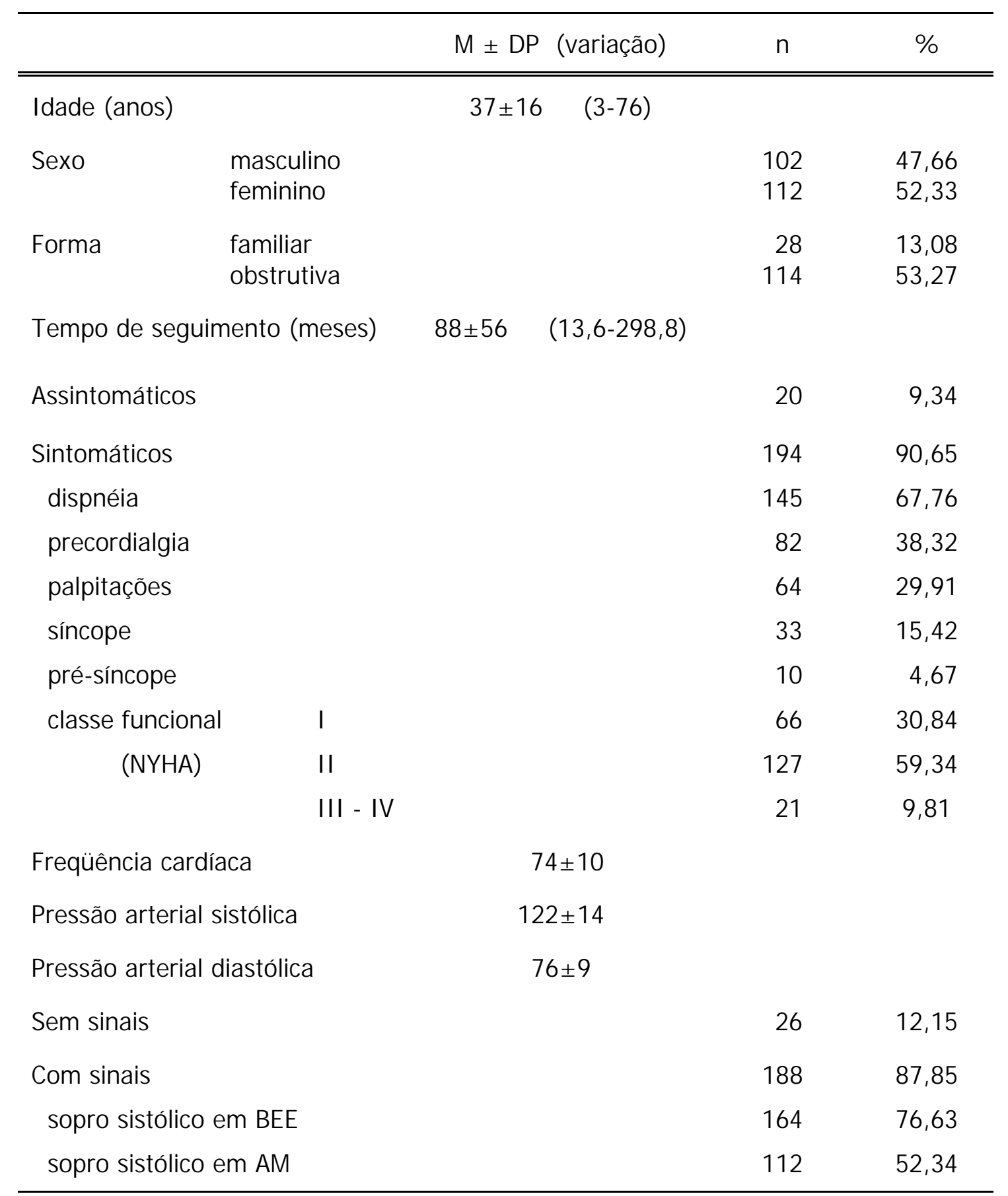

$\mathrm{AM}=$ área mitral; $\mathrm{BEE}=$ borda esternal esquerda; $\mathrm{M} \pm \mathrm{DP}=$ média \pm desvio padrão; $\mathrm{n}=$ número de pacientes; $\mathrm{NYHA}$ $=$ New York Heart Association 
TABELA 2. DROGAS UTILIZADAS EM PACIENTES PORTADORES DE CARDIOMIOPATIA HIPERTRÓFICA ( $\mathrm{N}=203$ )

\begin{tabular}{lccc}
\hline DROGAS & DOSE MÉDIA (variação) & $\mathrm{n}$ & $\%$ \\
\hline \hline Bloqueadoras de receptores $\beta$-adrenérgicos & 115 & 56,65 \\
$\begin{array}{l}\text { propranolol } \\
\text { atenolol }\end{array}$ & $280 \mathrm{mg} \quad(80-480)$ & 94 & 46,30 \\
nadolol & $50 \mathrm{mg} \quad(25-100)$ & 12 & 5,91 \\
metoprolol & $120 \mathrm{mg} \quad(80-160)$ & 5 & 2,46 \\
sotalol & $150 \mathrm{mg} \quad(100-200)$ & 2 & 0,98 \\
Bloqueadoras de canais de cálcio & $160 \mathrm{mg}$ & 2 & 0,98 \\
verapamil & $280 \mathrm{mg} \quad(80-480)$ & 76 & 37,44 \\
nifedipina & $40 \mathrm{mg}$ & 74 & 36,45 \\
Amiodarona & $400 \mathrm{mg} \quad(200-600)$ & 2 & 0,98 \\
Disopiramida & $250-500 \mathrm{mg}$ & 38 & 18,72 \\
\hline
\end{tabular}

$\mathrm{n}=$ número de pacientes 
TABELA 3. COMPARAÇÃO DAS VARIÁVEIS CLÍNICAS ENTRE 192 PACIENTES VIVOS E 22 PACIENTES QUE EVOLUÍRAM PARA ÓBITO

\begin{tabular}{|c|c|c|c|c|c|c|c|c|c|c|}
\hline \multirow{2}{*}{ Variável } & \multicolumn{4}{|c|}{ VIVOS } & \multicolumn{4}{|c|}{ ÓBITOS } & \multirow[b]{2}{*}{ TOTAL } & \multirow[b]{2}{*}{$p$} \\
\hline & $\mathrm{n}$ & $M$ & DP & $\%$ & $\mathrm{n}$ & $M$ & $\mathrm{DP}$ & $\%$ & & \\
\hline I dade & 192 & 36,87 & 15,96 & & 22 & 34,45 & 15,88 & & & 0,502 \\
\hline$\leq 20$ & 32 & - & - & 88,89 & 4 & & & 11,11 & 36 & 0,770 \\
\hline$>20$ & 160 & - & - & 89,89 & 18 & & & 10,11 & 178 & \\
\hline \multicolumn{11}{|l|}{ Sexo } \\
\hline feminino & 103 & - & - & 91,96 & 9 & & & 8,04 & 112 & 0,270 \\
\hline masculino & 89 & - & - & 87,25 & 13 & & & 12,75 & 102 & \\
\hline Forma & & & & & & & & & 214 & \\
\hline não familiar & 169 & - & & 90,86 & 17 & & & 9,14 & 186 & 0,179 \\
\hline familiar & 23 & - & & 82,14 & 5 & & & 17,86 & 28 & \\
\hline Forma & & & & & & & & & 214 & \\
\hline não obstrutiva & 31 & & & 16,15 & 1 & & & 4,55 & 31 & 0,211 \\
\hline obstrutiva & 161 & & & 83,85 & 21 & & & 95,45 & 182 & \\
\hline \multicolumn{11}{|l|}{ Sintomas } \\
\hline dispnéia & & & & & & & & & & \\
\hline não & 62 & - & - & 89,87 & 7 & & & 10,14 & 69 & 1,000 \\
\hline $\operatorname{sim}$ & 130 & - & - & 89,66 & 15 & & & 10,34 & 145 & \\
\hline \multicolumn{11}{|l|}{ precordialgia } \\
\hline não & 118 & - & - & 89,39 & 14 & & & 10,61 & 132 & 1,000 \\
\hline $\operatorname{sim}$ & 74 & - & - & 90,24 & 8 & & & 9,76 & 82 & \\
\hline \multicolumn{11}{|l|}{ palpitação } \\
\hline não & 139 & - & - & 92,67 & 11 & & & 7,33 & 150 & 0,047 \\
\hline sim & 53 & - & - & 82,81 & 11 & & & 17,19 & 64 & \\
\hline \multicolumn{11}{|l|}{ síncope } \\
\hline não & 161 & - & - & 88,95 & 20 & & & 11,05 & 181 & 0,5 \\
\hline $\operatorname{sim}$ & 31 & - & - & 93,94 & 2 & & & 6,06 & 33 & \\
\hline \multicolumn{11}{|l|}{ pré-síncope } \\
\hline não & 185 & - & - & 90,69 & 19 & & & 9,31 & 204 & 0,070 \\
\hline $\operatorname{sim}$ & 7 & - & - & 70,00 & 3 & & & 30,00 & 10 & \\
\hline \multicolumn{11}{|l|}{ Sinais } \\
\hline $\mathrm{FC}$ & 192 & 74,32 & 10,14 & & 22 & 74,32 & 10,88 & & & 0,986 \\
\hline PAS & 192 & 121,82 & 14,15 & & 22 & 125,23 & 10,74 & & & 0,276 \\
\hline PAD & 192 & 76,35 & 9,18 & & 22 & 77,73 & 9,35 & & & 0,508 \\
\hline \multicolumn{11}{|l|}{ SS BEE } \\
\hline não & 44 & - & - & 88,00 & 6 & & & 12,00 & 50 & 0,604 \\
\hline $\operatorname{sim}$ & 148 & - & - & 90,24 & 16 & & & 9,76 & 164 & \\
\hline \multicolumn{11}{|l|}{ SS AM } \\
\hline não & 92 & - & - & 90,20 & 10 & & & 9,80 & 102 & 1,000 \\
\hline $\operatorname{sim}$ & 100 & - & - & 89,29 & 12 & & & 10,71 & 112 & \\
\hline
\end{tabular}

$p=$ nível descritivo de probabilidade para o teste $t$ de Student e para o teste exato de Fisher

$\mathrm{DP}=$ desvio padrão; $\mathrm{FC}=$ freqüência cardíaca em batimentos por minuto; $M=$ média; $n=$ número de pacientes; $P A D$ = pressão arterial diastólica em milímetros de mercúrio; PAS = pressão arterial sistólica em milímetros de mercúrio; SS AM = sopro sistólico em área mitral; SS BEE = sopro sistólico em borda esternal esquerda 
TABELA 4. ESTUDO ELETROCARDIOGRÁFICO EM 214 PACIENTES PORTADORES DE CARDIOMIOPATIA HIPERTRÓFICA

\begin{tabular}{|c|c|c|}
\hline & $\mathrm{n}$ & $\%$ \\
\hline Normais & 26 & 12,15 \\
\hline Alterados & 188 & 87,85 \\
\hline sobrecarga do ventrículo esquerdo & 179 & 83,64 \\
\hline sobrecarga do átrio esquerdo & 36 & 16,82 \\
\hline fibrilação atrial & 18 & 8,41 \\
\hline síndrome de Wolff-Parkinson-White & 7 & 3,27 \\
\hline bloqueio do ramo esquerdo & 3 & 1,40 \\
\hline área eletricamente inativa & 3 & 1,40 \\
\hline sobrecarga do ventrículo direito & 2 & 0,93 \\
\hline
\end{tabular}

$\mathrm{n}=$ número de pacientes 
TABELA 5. COMPARAÇÃO DAS VARIÁVEIS ELETROCARDIOGRÁFICAS ENTRE 192 PACIENTES VIVOS E 22 PACIENTES QUE EVOLUÍRAM PARA ÓBITO

\begin{tabular}{|c|c|c|c|c|c|c|}
\hline \multirow[b]{2}{*}{ Variável } & \multicolumn{2}{|c|}{ VIVOS } & \multicolumn{2}{|c|}{ ÓBITOS } & \multirow[b]{2}{*}{ TOTAL } & \multirow[b]{2}{*}{$p$} \\
\hline & $\mathrm{n}$ & $\%$ & $\mathrm{n}$ & $\%$ & & \\
\hline \multicolumn{7}{|c|}{ Fibrilação atrial } \\
\hline não & 176 & 89,80 & 20 & 10,20 & 196 & 1,000 \\
\hline $\operatorname{sim}$ & 16 & 88,89 & 2 & 11,11 & 18 & \\
\hline \multicolumn{7}{|c|}{ Sobrecarga do átrio esquerdo } \\
\hline não & 160 & 89,89 & 18 & 10,11 & 178 & 0,770 \\
\hline $\operatorname{sim}$ & 32 & 88,89 & 4 & 11,11 & 36 & \\
\hline \multicolumn{7}{|c|}{ Sobrecarga do ventrículo esquerdo } \\
\hline não & 30 & 85,71 & 5 & 14,29 & 35 & 0,371 \\
\hline $\operatorname{sim}$ & 162 & 90,50 & 17 & 9,50 & 179 & \\
\hline
\end{tabular}

$p=$ nível descritivo de probabilidade para o teste exato de Fisher

$\mathrm{n}=$ número de pacientes 
TABELA 6. ELETROCARDIOGRAFIA DINÂMICA EM 172 PACIENTES PORTADORES DE CARDIOMIOPATIA HIPERTRÓFICA

\begin{tabular}{crr}
\hline & $\mathrm{n}$ & $\%$ \\
\hline \hline Sem arritmia & 19 & 11,04 \\
Com arritmia & 153 & 88,95 \\
supraventriculares & 98 & 56,97 \\
extrassístoles atriais & 92 & 53,49 \\
extrassístoles freqüentes (>30/hora) & 4 & 2,32 \\
taquicardia atrial paroxística & 1 & 0,58 \\
fibrilação atrial & 1 & 0,58 \\
ventriculares & 141 & 81,97 \\
extrassístoles freqüentes (>10/hora) & 34 & 19,76 \\
extrassístoles polimórficas & 35 & 20,35 \\
extrassístoles pareadas & 45 & 26,16 \\
taquicardia ventricular não sustentada & 33 & 19,18 \\
\hline
\end{tabular}

$\mathrm{n}=$ número de pacientes 
TABELA 7. COMPARAÇÃO DAS VARIÁVEIS OBTIDAS PELA ELETROCARDIOGRAFIA DINÂMICA EM PACIENTES VIVOS E EM PACIENTES QUE EVOLUÍRAM PARA ÓBITO

\begin{tabular}{|c|c|c|c|c|c|c|c|c|c|c|}
\hline \multirow[b]{2}{*}{ Variável } & \multicolumn{4}{|c|}{ VIVOS } & \multicolumn{4}{|c|}{ ÓBITOS } & \multirow[b]{2}{*}{ TOTAL } & \multirow[b]{2}{*}{$p$} \\
\hline & $\mathrm{n}$ & $M$ & $\mathrm{DP}$ & $\%$ & $n$ & $M$ & DP & $\%$ & & \\
\hline FCM & 158 & 72,13 & 11,40 & - & 14 & 72,00 & 10,31 & - & - & 0,968 \\
\hline ESV & 158 & 3,63 & 13,39 & - & 14 & 0,50 & 0,65 & - & - & 0,004 \\
\hline EV & 158 & 9,40 & 31,95 & - & 14 & 29,14 & 50,21 & - & - & 0,170 \\
\hline \multicolumn{11}{|l|}{ TVNS } \\
\hline não & 127 & - & - & 91,37 & 12 & - & - & 8,63 & 139 & 1,000 \\
\hline sim & 31 & - & - & 93,94 & 2 & - & - & 6,06 & 33 & \\
\hline
\end{tabular}

$p$ = nível descritivo de probabilidade para o teste $t$ de Student e para o teste exato de Fisher

DP = desvio padrão; ESV = extrassístoles supraventriculares; EV = extrassístoles ventriculares; FCM = freqüência cardíaca média em batimentos por minuto; $M$ = média; $n=$ número de pacientes; TVNS = taquicardia ventricular não sustentada 
TABELA 8. ESTUDO DA ECOCARDIOGRAFIA COM DOPPLER EM 214 PACIENTES PORTADORES DE CARDIOMIOPATIA HIPERTRÓFICA

\begin{tabular}{|c|c|c|c|}
\hline & $M \pm D P$ & $\mathrm{n}$ & $\%$ \\
\hline Espessura diastólica do septo interventricular (mm) & $21 \pm 5$ & - & - \\
\hline Espessura diastólica da parede livre do VE (mm) & $11 \pm 3$ & - & - \\
\hline Relação septo/parede & $1,93 \pm 0,60$ & - & - \\
\hline Tamanho do átrio esquerdo (mm) & $39 \pm 7$ & - & - \\
\hline Tamanho do VE em diástole (mm) & $42 \pm 6$ & - & - \\
\hline Gradiente da via de saída do VE (mmHg) & $47 \pm 30$ & - & - \\
\hline Fração de ejeção do ventrículo esquerdo & $0,79 \pm 0,07$ & - & - \\
\hline Movimento anterior sistólico da valva mitral & - & 146 & 68,22 \\
\hline Fechamento mesossistólico da valva aórtica & - & 120 & 56,07 \\
\hline Insuficiência da valva mitral & - & 83 & 38,78 \\
\hline
\end{tabular}

$\mathrm{M} \pm \mathrm{DP}=$ média \pm desvio padrão; $\mathrm{mm}=$ milímetros; $\mathrm{mmHg}=$ milímetros de mercúrio; $\mathrm{n}=$ número de pacientes; $\mathrm{VE}=$ ventrículo esquerdo 
TABELA 9. COMPARAÇÃO DAS VARIÁVEIS OBTIDAS PELA ECOCARDIOGRAFIA COM DOPPLER EM 192 PACIENTES VIVOS E EM 22 PACIENTES QUE EVOLUÍRAM PARA ÓBITO

\begin{tabular}{|c|c|c|c|c|c|c|c|c|c|c|}
\hline \multirow[b]{2}{*}{ Variável } & \multicolumn{4}{|c|}{ VIVOS } & \multicolumn{4}{|c|}{ ÓBITOS } & \multirow[b]{2}{*}{ TOTAL } & \multirow[b]{2}{*}{$\mathrm{p}$} \\
\hline & $n$ & $M$ & $\mathrm{DP}$ & $\%$ & $n$ & $M$ & $\mathrm{DP}$ & $\%$ & & \\
\hline Septo & 192 & 20,52 & 4,81 & - & 22 & 21,09 & 4,12 & - & - & 0,591 \\
\hline Parede livre & 192 & 11,17 & 3,44 & - & 22 & 11,55 & 2,50 & - & - & 0,617 \\
\hline Septo/parede & 192 & 1,93 & 0,61 & & 22 & 1,87 & 0,55 & - & - & 0,622 \\
\hline Átrio esquerdo & 188 & 39,10 & 7,40 & & 22 & 38,23 & 7,48 & - & - & 0,603 \\
\hline VE & 187 & 42,15 & 5,71 & & 21 & 42,86 & 6,76 & - & - & 0,598 \\
\hline FE & 185 & 0,79 & 0,07 & & 20 & 0,80 & 0,08 & - & - & 0,470 \\
\hline Gradiente VSVE & 91 & 47,47 & 29,53 & & 7 & 45,86 & 37,80 & - & - & 0,892 \\
\hline \multicolumn{11}{|l|}{ MASVM } \\
\hline não & 65 & & & 95,59 & 3 & & & 4,41 & 68 & 0,057 \\
\hline $\operatorname{sim}$ & 127 & & & 86,99 & 19 & & & 13,01 & 146 & \\
\hline \multicolumn{11}{|l|}{ FMSVA } \\
\hline não & 89 & & & 94,68 & 5 & & & 5,32 & 94 & 0,041 \\
\hline $\operatorname{sim}$ & 103 & & & 89,83 & 17 & & & 14,17 & 120 & \\
\hline \multicolumn{11}{|l|}{$\mid \mathrm{Ml}$} \\
\hline não & 117 & & & 89,31 & 14 & & & 10,69 & 131 & 1,000 \\
\hline $\operatorname{sim}$ & 75 & & & 90,36 & 8 & & & 9,64 & 83 & \\
\hline
\end{tabular}

$p=$ nível descritivo de probabilidade para o teste $t$ de Student e para o teste exato de Fisher

átrio esquerdo = tamanho do átrio esquerdo em milímetros; DP = desvio padrão; FE = fração de ejeção do ventrículo esquerdo; FMSVA = fechamento mesossistólico da valva aórtica; gradiente VSVE = gradiente da via de saída do ventrículo esquerdo em milímetros de mercúrio; IMI = insuficiência da valva mitral; $\quad$ MASVM = movimento anterior sistólico da valva mitral; $\quad M=$ média; $n=$ número de pacientes; parede livre = espessura diastólica da parede livre do ventrículo esquerdo em milímetros; septo = espessura diastólica do septo interventricular em milímetros; septo/parede = relação septo/parede; VE = tamanho do ventrículo esquerdo em diástole em milímetros 
TABELA 10. ESTUDO HEMODINÂMICO EM 50 PACIENTES PORTADORES DE CARDIOMIOPATIA HIPERTRÓFICA

\begin{tabular}{lcc}
\hline \hline & $M \pm D P$ & VARIAÇÃO \\
\hline Pressão na ponta do ventrículo esquerdo $(\mathrm{mmHg})$ & $168 \pm 44$ & - \\
Pressão na via de saída do ventrículo esquerdo $(\mathrm{mmHg})$ & $125 \pm 29$ & $30-150$ \\
\hline
\end{tabular}

$\mathrm{M} \pm \mathrm{DP}=$ média \pm desvio padrão; $\mathrm{mmHg}=$ milímetros de mercúrio 
TABELA 11. COMPARAÇÃO DAS VARIÁVEIS HEMODINÂMICAS OBTIDAS EM PACIENTES VIVOS E EM PACIENTES QUE EVOLUÍRAM PARA ÓBITO

\begin{tabular}{|c|c|c|c|c|c|c|c|}
\hline \multirow[b]{2}{*}{ Variável } & \multicolumn{3}{|c|}{ VIVOS } & \multicolumn{3}{|c|}{ ÓBITOS } & \multirow[b]{2}{*}{$\mathrm{p}$} \\
\hline & $\mathrm{n}$ & M & DP & $\mathrm{n}$ & M & DP & \\
\hline Pressão na ponta do VE & 39 & 166,62 & 45,42 & 9 & 171,89 & 39,90 & 0,750 \\
\hline Pressão na via de saída do VE & 39 & 126,36 & 28,51 & 9 & 118,67 & 31,05 & 0,476 \\
\hline Gradiente da via de saída do VE & 41 & 41,05 & 37,37 & 9 & 53,22 & 35,87 & 0,378 \\
\hline
\end{tabular}

$p=$ nível descritivo de probabilidade para $o$ teste $t$ de Student

$\mathrm{DP}=$ desvio padrão; $\mathrm{M}=$ média; $\mathrm{n}=$ número de pacientes; $\mathrm{VE}=$ ventrículo esquerdo 
TABELA 12. TRATAMENTO E RESPOSTA OBTIDAS EM 192 PACIENTES VIVOS E EM 22 PACIENTES QUE EVOLUÍRAM PARA ÓBITO

\begin{tabular}{|c|c|c|c|c|c|c|}
\hline \multirow[b]{2}{*}{ Variável } & \multicolumn{2}{|c|}{ VIVOS } & \multicolumn{2}{|c|}{ ÓBITOS } & \multirow[b]{2}{*}{ TOTAL } & \multirow[b]{2}{*}{$p$} \\
\hline & $n$ & $\%$ & $n$ & $\%$ & & \\
\hline \multicolumn{7}{|l|}{ Tratamento } \\
\hline clínico & 165 & 89,19 & 20 & 10,81 & 185 & 0,538 \\
\hline cirúrgico & 14 & 87,50 & 2 & 12,50 & 16 & \\
\hline marcapasso & 13 & 100,00 & 0 & 0,00 & 13 & \\
\hline \multicolumn{7}{|l|}{ Resposta } \\
\hline melhorado & 158 & 94,05 & 10 & 5,95 & 168 & $<0,001$ \\
\hline estável & 34 & 82,93 & 7 & 17,07 & 41 & \\
\hline piorado & 0 & 0,00 & 5 & 100,00 & 5 & \\
\hline \multicolumn{7}{|l|}{ Medicação } \\
\hline \multicolumn{7}{|l|}{ propranolol } \\
\hline não & 110 & 91,67 & 10 & 8,33 & 120 & 0,365 \\
\hline $\operatorname{sim}$ & 82 & 87,23 & 12 & 12,77 & 94 & \\
\hline \multicolumn{7}{|l|}{ verapamil } \\
\hline não & 124 & 88,57 & 16 & 11,43 & 140 & 0,490 \\
\hline $\operatorname{sim}$ & 68 & 91,89 & 6 & 8,11 & 74 & \\
\hline \multicolumn{7}{|l|}{ amiodarona } \\
\hline não & 159 & 90,34 & 17 & 9,66 & 176 & 0,556 \\
\hline $\operatorname{sim}$ & 33 & 86,84 & 5 & 13,16 & 38 & \\
\hline
\end{tabular}

$p=$ nível descritivo de probabilidade para o teste exato de Fisher

$\mathrm{n}=$ número de pacientes 
TABELA 13. CARACTERIZAÇÃO CLÍNICA DE 22 PACIENTES PORTADORES DE CARDIOMIOPATIA HIPERTRÓFICA QUE EVOLUÍRAM PARA ÓBITO

\begin{tabular}{|c|c|c|c|c|c|c|c|c|c|c|c|c|c|c|c|c|}
\hline \multirow[t]{2}{*}{ № } & \multirow{2}{*}{$\begin{array}{l}\text { Idade } \\
\text { (anos) }\end{array}$} & \multirow[t]{2}{*}{ Sexo } & \multirow{2}{*}{$\begin{array}{c}\text { Tempo de } \\
\text { Seguimento } \\
\text { (meses) }\end{array}$} & \multirow[t]{2}{*}{ Forma } & \multirow{2}{*}{$\begin{array}{c}\text { CF } \\
\text { inicial }\end{array}$} & \multirow{2}{*}{$\begin{array}{c}\text { ECG } \\
\text { FA }\end{array}$} & \multirow{2}{*}{$\begin{array}{l}\text { ECGD } \\
\text { TVNS }\end{array}$} & \multicolumn{3}{|c|}{ ECO } & \multirow{2}{*}{$\begin{array}{l}\text { Hemo } \\
\text { Grad. }\end{array}$} & \multicolumn{2}{|c|}{ Tratamento } & \multirow[t]{2}{*}{ Forma } & \multirow{2}{*}{$\begin{array}{c}\text { CF } \\
\text { final }\end{array}$} & \multirow[t]{2}{*}{ Causa } \\
\hline & & & & & & & & Septo & $\mathrm{AE}$ & Grad. & & Clin. & Cir. & & & \\
\hline 1 & 25 & $\bar{M}$ & 22,2 & $\mathrm{~N}$ & $\bar{I}$ & A & 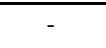 & 30 & 26 & 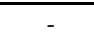 & 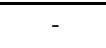 & $\bar{S}$ & $\mathrm{~N}$ & $\mathrm{O}$ & $\bar{I}$ & MS \\
\hline 2 & 49 & $\mathrm{~F}$ & 116,9 & $\mathrm{~N}$ & III & A & $P$ & 18 & 50 & 30 & 36 & S & $\mathrm{N}$ & $\mathrm{O}$ & 1 & AVC \\
\hline 3 & 27 & $\mathrm{~F}$ & 158,7 & $\mathrm{~F}$ & II & $A$ & $A$ & 20 & 40 & 30 & - & $\mathrm{s}$ & $N$ & $\mathrm{O}$ & I & MS \\
\hline 4 & 51 & $\mathrm{~F}$ & 29,1 & $\mathrm{~N}$ & III & $P$ & $A$ & 18 & 44 & 120 & 75 & S & $N$ & $\mathrm{O}$ & IV & ICC \\
\hline 5 & 56 & $M$ & 11,2 & $\mathrm{~N}$ & II & $A$ & - & 17 & 41 & 71 & - & S & $\mathrm{N}$ & $\mathrm{O}$ & 1 & $\mathrm{CH}$ \\
\hline 6 & 43 & M & 33,0 & $\mathrm{~N}$ & II & A & - & 28 & 43 & - & - & $\mathrm{s}$ & $\mathrm{N}$ & $\mathrm{O}$ & I & acidental \\
\hline 7 & 18 & $\mathrm{~F}$ & 73,6 & $\mathrm{~F}$ & 1 & A & A & 20 & 38 & - & 73 & $\mathrm{~S}$ & $\mathrm{~N}$ & $\mathrm{O}$ & 1 & MS \\
\hline 8 & 61 & $M$ & 123,8 & $\mathrm{~N}$ & II & A & $P$ & 24 & 40 & - & - & $S$ & $\mathrm{~N}$ & $\mathrm{O}$ & 1 & NAD \\
\hline 9 & 39 & $\mathrm{~F}$ & 84,2 & $\mathrm{~N}$ & II & A & A & 23 & 27 & - & - & $\mathrm{s}$ & $\mathrm{N}$ & NO & I & pneumonia \\
\hline 10 & 46 & $\mathrm{~F}$ & 5,4 & $\mathrm{~F}$ & II & A & - & 17 & 46 & - & - & $\mathrm{s}$ & $\mathrm{N}$ & $\mathrm{O}$ & 1 & MS \\
\hline 11 & 13 & $M$ & 13,0 & $N$ & 1 & A & A & 21 & 33 & - & - & $\mathrm{s}$ & $\mathrm{N}$ & $\mathrm{O}$ & 1 & MS \\
\hline 12 & 16 & $M$ & 0,6 & $\mathrm{~F}$ & IV & A & - & 23 & 54 & 38 & - & $\mathrm{s}$ & $\mathrm{N}$ & $\mathrm{O}$ & IV & MS \\
\hline 13 & 48 & $M$ & 96,1 & $\mathrm{~N}$ & 1 & $P$ & - & 23 & 36 & - & 53 & $\mathrm{~S}$ & $\mathrm{~N}$ & $\mathrm{O}$ & 1 & MS \\
\hline 14 & 32 & $M$ & 47,6 & $\mathrm{~N}$ & II & A & A & 25 & 38 & - & 70 & $\mathrm{~s}$ & $\mathrm{~N}$ & $\mathrm{O}$ & 11 & MS \\
\hline 15 & 3 & $M$ & 120,7 & $\mathrm{~N}$ & 1 & A & - & 18 & 30 & - & - & $\mathrm{s}$ & $\mathrm{N}$ & $\mathrm{O}$ & 1 & acidental \\
\hline 16 & 63 & $\mathrm{~F}$ & 44,0 & $N$ & II & A & - & 21 & 47 & - & 122 & $\mathrm{~s}$ & $\mathrm{~S}$ & $\mathrm{O}$ & IV & ICC \\
\hline 17 & 28 & $M$ & 83,9 & $N$ & 1 & A & A & 15 & 39 & - & - & $\mathrm{s}$ & $\mathrm{N}$ & $\mathrm{O}$ & 1 & IRC \\
\hline 18 & 33 & $M$ & 117,4 & $\mathrm{~F}$ & II & A & A & 20 & 33 & - & - & $\mathrm{s}$ & $N$ & $\mathrm{O}$ & 1 & acidental \\
\hline 19 & 28 & $\mathrm{~F}$ & 37,8 & $N$ & III & A & A & 16 & 28 & - & - & $\mathrm{s}$ & $\mathrm{S}$ & $\mathrm{O}$ & III & MS \\
\hline 20 & 26 & $\mathrm{~F}$ & 37,7 & $\mathrm{~N}$ & II & A & A & 17 & 38 & - & - & $\mathrm{S}$ & $\mathrm{N}$ & $\mathrm{O}$ & 11 & El \\
\hline 21 & 31 & $M$ & 50,0 & $N$ & 1 & A & A & 22 & 40 & - & - & $\mathrm{s}$ & $\mathrm{N}$ & $\mathrm{O}$ & 1 & MS \\
\hline 22 & 26 & $M$ & 189,8 & $\mathrm{~N}$ & II & A & A & 28 & 30 & - & - & $\mathrm{s}$ & $\mathrm{N}$ & $\mathrm{O}$ & 111 & MS \\
\hline
\end{tabular}

$\mathrm{A}=$ ausente; $\mathrm{AE}=$ átrio esquerdo; $\mathrm{AVC}=$ acidente vascular cerebral; $\mathrm{CF} \mathrm{NYHA}=$ classificação funcional segundo critérios da New York Heart Association, $\mathrm{CH}=\mathrm{cirrose}$ hepática; Cir. = cirúrgico; Clin. = clínico; ECG = eletrocardiograma; ECGD = eletrocardiograma dinâmico com registro de 24 horas; ECO = ecocardiograma; El = endocardite infecciosa; FA = fibrilação atrial; Grad. = gradiente; Hemo = estudo hemodinâmico; ICC = insuficiência cardíaca congestiva; IRC = insuficiência renal crônica; MS $=$ morte súbita; NAD = neoplasia do aparelho digestivo; $\mathrm{P}=$ presente; TVNS = taquicardia ventricular não sustentada

sexo: $F=$ feminino; $M=$ masculino; forma: $F=$ familiar; $N=$ não familiar; $O=$ obstrutiva; $N O=$ não obstrutiva; tratamento: $S=$ sim; $N=$ não 
TABELA 14. EVOLUÇÃO E CAUSA DE ÓBITO EM 214 PACIENTES PORTADORES DE CARDIOMIOPATIA HIPERTRÓFICA

\begin{tabular}{|c|c|c|}
\hline PACIENTES & $n$ & $\%$ \\
\hline Vivos & 192 & 89,72 \\
\hline Óbitos & 22 & 10,28 \\
\hline Relacionados à doença & 14 & 6,54 \\
\hline morte súbita & 11 & 5,14 \\
\hline insuficiência cardíaca congestiva & 2 & 0,93 \\
\hline endocardite infecciosa & 1 & 0,46 \\
\hline Não relacionados com a doença & 8 & 3,74 \\
\hline acidental & 3 & 1,40 \\
\hline acidente vascular cerebral & 1 & 0,46 \\
\hline pneumonia & 1 & 0,46 \\
\hline cirrose hepática & 1 & 0,46 \\
\hline neoplasia do aparelho digestivo & 1 & 0,46 \\
\hline insuficiência renal crônica & 1 & 0,46 \\
\hline
\end{tabular}

$\mathrm{n}$ = número de pacientes 
TABELA 15. CARDIOMIOPATIA HIPERTRÓFICA - COMPARAÇÃO ENTRE OS ACHADOS CLÍNICOS E SOBREVIDA

\begin{tabular}{|c|c|c|c|c|c|c|c|c|c|}
\hline Autores - País/ano & $\mathrm{n}$ & $\begin{array}{l}\text { Tempo de } \\
\text { Seguimento } \\
\text { (anos) }\end{array}$ & $\begin{array}{l}\text { Idade } \\
\text { (anos) }\end{array}$ & $\begin{array}{c}\text { Forma } \\
\text { Familiar } \\
\%\end{array}$ & $\begin{array}{c}\text { CF-NYHA } \\
\text { III-IV } \\
\%\end{array}$ & $\begin{array}{c}\text { Síncope } \\
\%\end{array}$ & $\begin{array}{l}\text { FA } \\
\%\end{array}$ & $\begin{array}{c}\text { TVNS } \\
\%\end{array}$ & Septo \\
\hline Frank S. et al. - EUA/1968 & 126 & 12 & - & - & 22 & - & 8 & & - \\
\hline Shah P. et al. - EUA-RU-C/1974 & 190 & 5,2 & - & 20 & 36 & 27 & 7 & & - \\
\hline McKenna W. et al. - RU/1981 & 254 & 6 & 34 & 24 & 22 & 49 & 15 & & - \\
\hline Shapiro LM. et al-RU/1983 & 39 & 3,1 & 55 & 36 & 20 & 23 & 15 & 5 & - \\
\hline Koga Y. et al. - Japão/1984 & 136 & 5,1 & 38 & - & - & - & 11 & - & - \\
\hline Spirito P. et al. - I talia/1989 & 25 & 4,4 & 44 & - & 4 & - & - & 24 & 20 \\
\hline Romeo F. et al. - I talia/1990 & 125 & 7,6 & 34 & 23 & 65 & 30 & 7 & 16 & - \\
\hline Fay WP. et al. - EUA/1990 & 95 & 4,2 & 71 & - & 13 & 17 & - & - & 20 \\
\hline Seiler C. et al. - Suiça/1991 & 139 & 8,9 & 37 & - & - & - & 3 & - & - \\
\hline Kofflard MJ . et al. - Holanda/1993 & 113 & 7 & 37 & 48 & 14 & 21 & - & 32 & - \\
\hline Azzano O. et al. - França/1995 & 40 & 10,2 & 10.9 & 53 & 32 & 17 & - & - & - \\
\hline Cannan CR. et al. - EUA/1995 & 37 & 7,7 & 59 & - & 11 & 11 & - & - & 17,5 \\
\hline Cecchi F. et al. - Italia/1995 & 202 & 10,1 & 41 & 43 & 9 & 12 & 28 & 25 & 23 \\
\hline Maron BJ . et al. - EUA/1996 & 227 & 8 & 45 & - & - & - & - & - & - \\
\hline
\end{tabular}

$\mathrm{n}=$ número de casos

* crianças; \# adultos; + clínicos; § cirúrgicos 


\section{Gráficos}

GRÁFICO 1. PACIENTES PORTADORES DE CARDIOMIOPATIA HIPERTRÓFICA: DISTRIBUIÇÃO POR FAIXA ETÁRIA

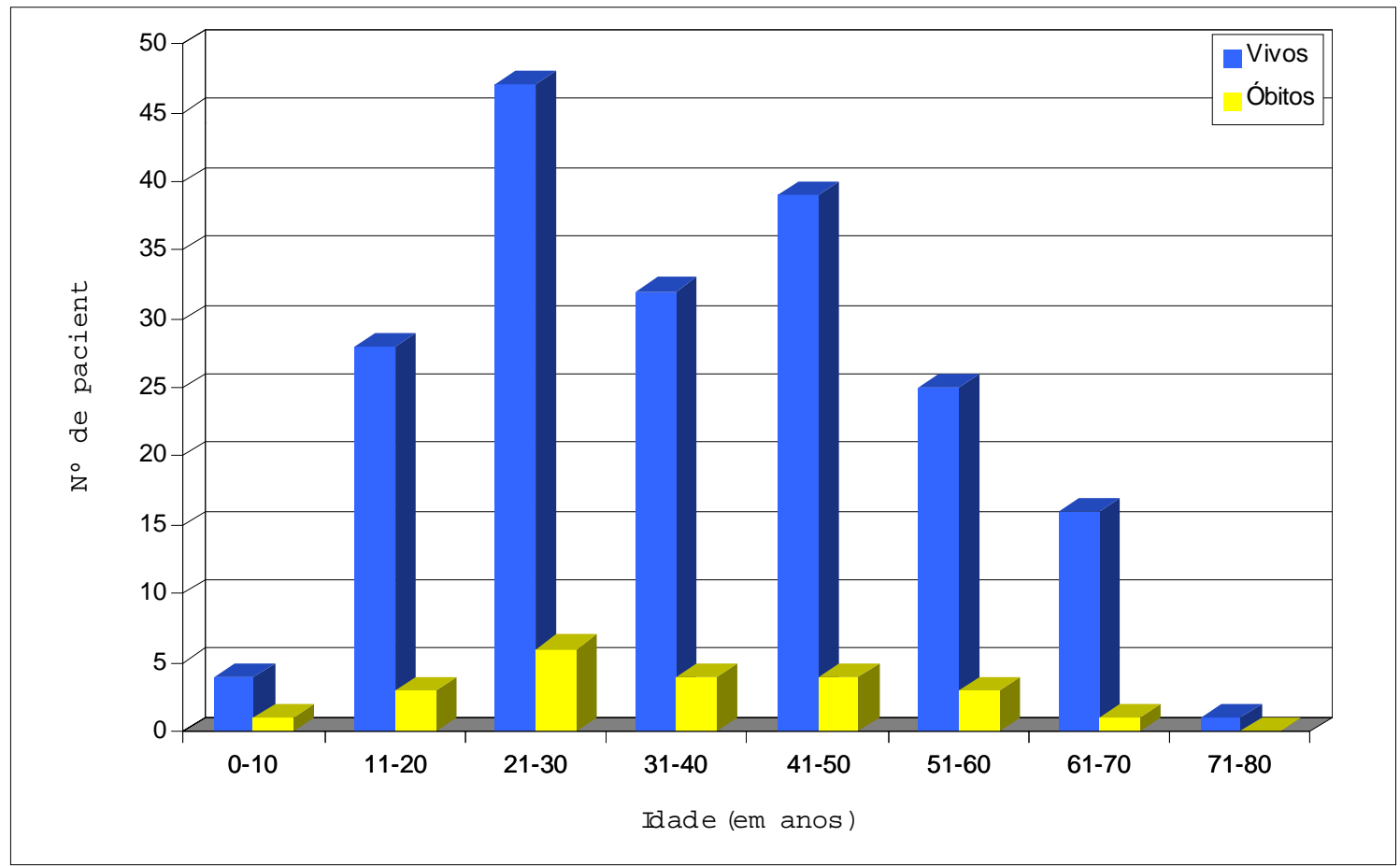


GRÁFICO 2. PACIENTES PORTADORES DE CARDIOMIOPATIA HIPERTRÓFICA: DISTRIBUIÇÃO POR TEMPO DE SEGUIMENTO

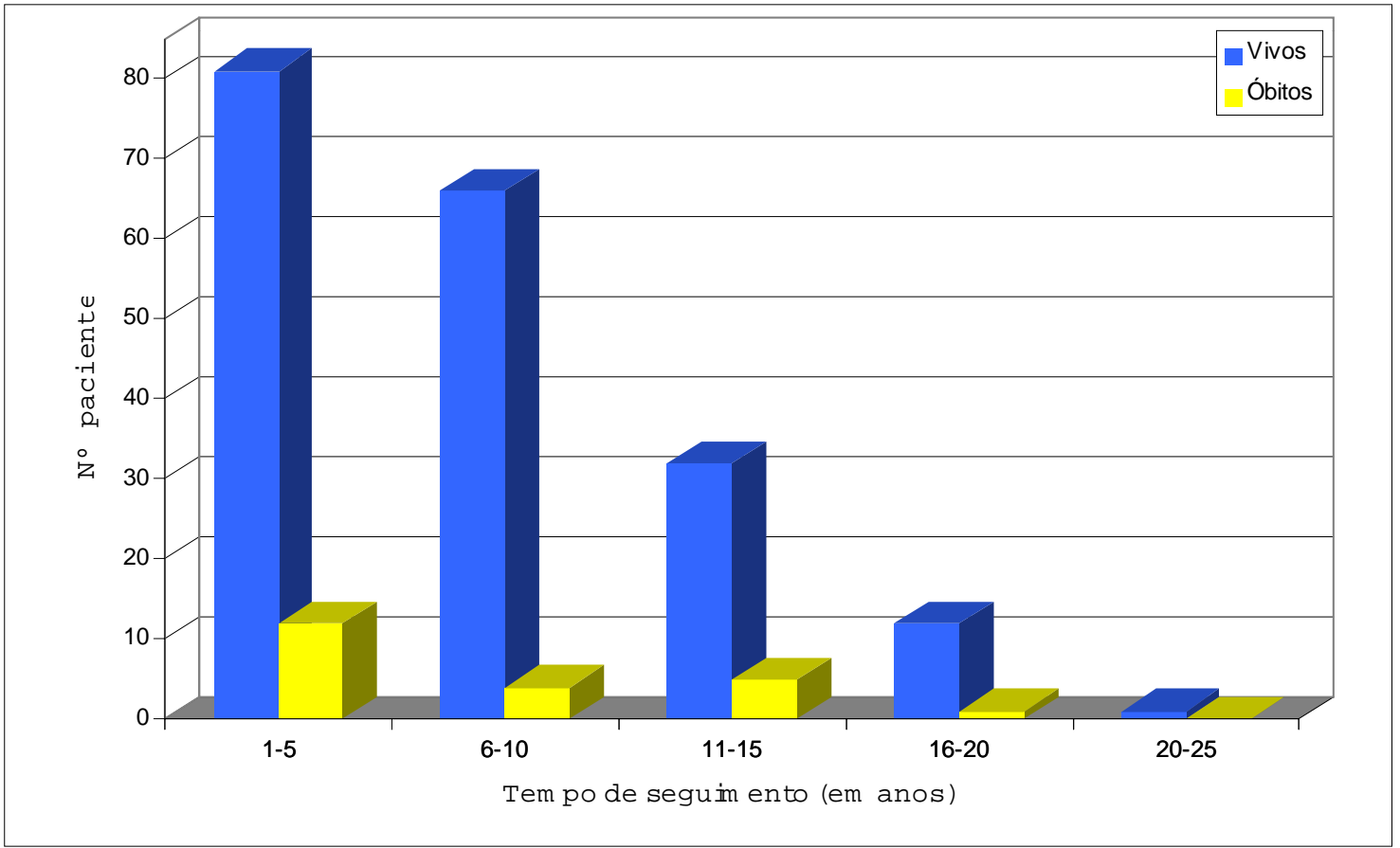




\section{REFERÊNCIAS}

1. TEARE, D. Asymetrical hypertrophy of the heart in young adults. $\mathrm{Br}$ HeartJ 20:1-8, 1958.

2. GOODWIN, J.F.; HOLLMAN, A.; CLELAND, W.P.; TEARE, D. Obstructive cardiomyopathy simulating aortic stenosis. Br Heart J 22:403-414, 1960.

3. BRAUNWALD, E.; LAMBREW, C.T.; ROCKOFF, S.D.; ROSS, J., J r.; MORROW, A.C. Idiopathic hypertrophic subaortic stenosis. 1. A description of the disease based upon an analysis of 64 patients. Circulation 30(Suppl. IV):IV3-IV119, 1964.

4. MARON, B.J.; BONOW, R.O.; CANNON III, R.O.; LEON, M.B.; EPSTEIN, S.E. Hypertrophic cardiomyopathy: Interrelations of clinical manifestations, pathophysiology, and therapy (two parts). N Engl J Med 316(13): 780-789, 1987; 316(14):844-852.

5. WIGLE, E.D.; SASSON, Z.; HENDERSON, M.A.; RUDDY, T.D.; FULOP, J.; RACOWSKI, H.; WILLIAMS, W.G. Hypertrophic cardiomyopathy. The importance of the site and the extent of hypertrophy. A review. Progr Cardiovasc Dis 28(1):183, 1985.

6. MARON, B.J. Hypertrophic cardiomyopathy. Curr Probl Cardiol 18:639-704, 1993.

7. MARON, B.J. Hypertrophic cardiomyopathy. Lancet 350:127-133, 1997.

8. MARON, B.J.; GARDIN, J.M.; FLACK, J.M.; GIDDING, S.S.; KUROSAKI, T.T.; BILD, D.E. Prevalence of hypertrophic cardiomyopathy in a general population of young adults. Echocardiograpic analysis of 4111 subjects in the CARDIA Study. Coronary Artery Risk Development in (Young) Adults. Circulation 92(4): 785-789, 1995.

9. HALLOPEAU, M. Rétrécissement ventriculo-aortique. Gazette Med Paris 24:683$684,1869$.

10. LIOUVILLE, H. Rétrécissement cardiaque sous aortique. Gazette Med Paris 24:161-163,1869.

11. SCHMINCKE, A. Ueber linkseitige muskulöse Conusstenosen. Dtsch Med Wochenschr 33:2082, 1907.

12. DAVIES, L.G. Familial heart disease. Br Heart J 14:206-212, 1952.

13. BROCK, R. Functional obstruction of the left ventricle (acquired aortic subvalvular stenosis). Guy's Hosp Rep 106:221-238, 1957. 
14. WATKINS, H. Multiple disease gene cause hypertrophic cardiomyopathy. $\mathrm{Br}$ Heart J 72(Suppl.):S4-S9, 1994.

15. WATKINS, H.; ROSENWEIG, A.; HWANG, D-S.; LEVI, T.; MCKENNA, W; SEIDMAN, C.E.; SEIDMAN, J.G. Characteristics and prognostic implications of myosin missense mutations in familial hypertrophic cardiomyopathy. N Eng/J Med 326:1108-1114, 1992.

16. SPIRITO, P.; SEIDMAN, C.E.; MCKENNA, W.J.; MARON, B.J. The management of hypertrophic cardiomyopathy. N Eng/J Med 336:775-785, 1997.

17. MARON, B.J.; NICHOLS, P.F. III; PICKLE, L.W.; WESLEY, Y.E.; MULTINIHILL, J.J. Patterns of inheritance in hypertrophic cardiomyopathy: assessment by Mmode and two-dimensional echocardiography. Am J Cardiol 53:1087-1094, 1984.

18. MARON, B.J.; ANAN, T.J.; ROBERTS, W.C. Quantitative analysis of the distribution of cardiac muscle cell disorganization in the left ventricular wall of patients with hypertrophic cardiomyopathy. Circulation 63:882-894, 1981.

19. KLUES, H.G.; MARON, B.J.; DOLLAR, A.L.; ROBERTS, W.C. Diversity of structural mitral valve alterations in hypertrophic cardiomyopathy. Circulation 85:16511660, 1992.

20. MARON, B.J.; WOLFSON, J.K; EPSTEIN, S.E.; ROBERTS, W.C. Intramural ("Small vessel") coronary artery disease in hypertrophic cardiomyopathy. J Am Coll Cardio/ 8:545-557, 1986.

21. FACTOR, S.M.; BUTANY, J.; SOLE, M.J.; WIGEL, E.D.; WILLIANS, W.C.; ROJ KIND, M. Pathologic fibrosis and matrix connective tissue in the subaortic myocardium of patients with hypertrophic cardiomyopathy. J Am Coll Cardiol 17:1343-1351, 1991.

22. TANAKA, M.; FUJIWARA, H.; ONODERA, T.; WU, N.J.; HAMASHIMA, Y.; KAWAI, C. Quantitative analysis of myocardial fibrosis in normal, hypertensive hearts, and hypertrophic cardiomyopathy. Br HeartJ 55:575-581, 1986.

23. MARON, B.J.; GOTTDIENER, J.S.; EPSTEIN, S.E. Patterns and significance of distribution of left ventricular hypertrophy in hypertrophic cardiomyopathy; a wide-angle, two-dimensional echocardiographic study of 125 patients. Am J Cardiol 48:418-428, 1981.

24. MARON, B.J.; ROBERTS, W.C.; EPSTEIN, S.E. Sudden death in hypertrophic cardiomyopathy: a profile of 78 patients. Circulation 67:1388-1394, 1982.

25. SPIRITO, P.; BELLONI, P. Natural history of hypertrophic cardiomyopathy. $\mathrm{Br}$ Heart J 72:S10-S12, 1994. 
26. SHAH, P.M.; ADELMAN, A.G.; WIGLE, E.D. , GOBEL, F.L.; BURCHELL, H.B.; HARDARSON, M.D.; CURIEL, M.D.; CALZADA, C.; OAKLEY, C.M.; GOODWIN, J.F. The natural (and unnatural) history of hypertrophic obstructive cardiomyopathy. Circ Res 34 and 35(Suppl 2):II179-195, 1974.

27. MCKENNA, W.J.; DEANFIELD, J.E.; FARUQUI, A.; ENGLAND, D.; OAKLEY, C.; GOODWIN, J. Prognosis in hypertrophic cardiomyopathy: role of age and clinical, electrocardiographic and hemodynamic features. Am J Cardiol 47:532-538, 1981.

28. KOGA, Y.; ITAYA, K-I.; TOSHIMA, H. Prognosis in hypertrophic cardiomyopathy. Am Heart J 108:351-359, 1984.

29. ROMEO, F.; PELLICCIA, F.; CRISTOFANI, R.; MARTUCELLI, E.; REALE, A. Hypertrophic cardiomyopathy: is a left ventricular outflow tract gradient a major prognostic determinant? Eur Heart J 11:233-240, 1990.

30. FAY, W.P.; TALIERCIO,C.P.; ILSTRUP, D.M.; TAJIK, A.J.; GERSH, B.J. Natural history of hypertrophic cardiomyopathy in the elderly. J Am Coll Cardiol 16:821826, 1990.

31. SEILER, C.; HESS, O.M.; SCHOENBECK, M.; TURINA, J.; JENNI, R.; TURINA, M.; KRAYENBUEHL, H-P. Long-term follow-up of medical versus surgical therapy for hypertrophic cardiomyopathy: a retrospective study. J Am Coll Cardiol 17:634642, 1991.

32. SHAPIRO, L.M.; ZEZULKA, A. Hypertrophic cardiomyopathy: a common disease with a good prognosis - five year experience of a district general hospital. $\mathrm{Br}$ Heart J 50:530-533, 1983.

33. SPIRITO, P.; CHIARELLA, F.; CARRATINO, L.; BERISSO, M.Z.; BELLOTTI, P.; VECCHIO, C. Clinical course and prognosis of hypertrophic cardiomyopathy in an outpatient population. N Eng/J Med 320:749-755, 1989.

34. KOFLARD, M.J.; WALDSTEIN, D.J.; VOS, J.; ten CATE, F.J. Prognosis in hypertrophic cardiomyopathy observed in a large clinic population. Am I Cardiol 72:939-943, 1993.

35. CANNAN, C.R.; REEDER, G.S.; BAILEY K.R.; MELTON III, J.M; GERSH, B.J . Natural history of hypertrophic cardiomyopathy - a population-based study, 1976 through 1990. Circulation 92:2488-2495, 1995.

36. CECCHI, F.; OLIVOTTO, I.; MONTEREGGI, A.; SANTORO, G.; DOLARA, A.; MARON, B.J. Hypertrophic cardiomyopathy in Tuscany: clinical course and outcome in an unselected regional population. J Am Coll Cardiol 26:1529-1536, 1995. 
37. MARON, B.J.; PLIAC, L.C.; CASEY, S.A.; LANGE, S.K.; AEPPLI, D. Clinical significance and consequences of hypertrophic cardiomyopathy assessed in an unselected patient population: evidence for the relatively benign nature of the true disease state in adulthood. Circulation 94(Suppl I):I-84, 1996.

38. ARTEAGA, E.; BARRETTO, A.C.P.; MADY, C.; IANNI B.M.; HOSNI, J.J.; BELLOTTI,G. Hypertrophic cardiomyopathy: a disease of a good prognosis despite the high incidence of ventricular arrhythmias. Circulation 92(8):I-98, 1995.

39. ARTEAGA, E.; PEREIRA BARRETTO, A.C.; IANNI, B.M.; MADY, C.; BELLOTTI, G.; PILEGGI, F. Cardiomiopatia hipertrófica em gerontes: dados clínicos e evolutivos. Rev Soc Cardiol Estado de São Paulo 3:63-68, 1991.

40. MARON, B.J .; SPIRITO, P. Impact of patient selection biases on the perception of hypertrophic cardiomyopathy and its natural history. Am J Cardiol 72:970-972, 1993.

41. COOKEY, J.D., DUNN, M.; MASSIE, E. Clinical vectorcardiography and electrocardiography. 2.ed. Chicago, Year Book Medical Publishers, Inc., 1977.

42. SAHN, D.J.; DeMARIA, A.N.; KISSLO, J.; WEYMAN, A. The Committee on Mmode Standartization of the American Society of Echocardiography. Recommendations regarding quantitation in M-mode echocardiography: results of a survey of echocardiographic measurements. Circulation 58:1072-1083, 1978.

43. HENRY W.L.; DeMARIA, A.N.; GRAMIAK, R.; KING, D.L.; KISSLO, J.A.; POPP, R.L.; SAHN, D.J .; SCHILLER, N.B.; TAJIK, A.; FEICHHOLZ, L.E.; WEYMAN, A.E. Report of the American Society of Echocardiography Committee on Nomenclature and Standards in Two-dimensional Echocardiography. Circulation 62:212-217, 1980.

44. SCHILLER, N.B.; SHAN, P.M.; CRAWFORD, M.; DeMARIA, A.; DEVEREUX, R.; FEIGENBAUM, H.; GUTGESLL, H.; SCHNITTGER, I. American Society of Echocardiography Committee on Standards, subcommittee on quantification of two-dimensional echocardiograms. Recommendations for quantification of the left ventricle by two-dimensional echocardiography. J Am Echocardiography 2:358-367, 1989.

45. HATLE, L.; ANGELSEN, B. Doppler ultrasound in cardiology. Physical principles and clinical applications. Philadelphia, Lea \& Febiger, 1985.

46. POMBO, J.F.; TROY, B.L.; RUSSELL, Jr. R.O. Left ventricular volumes and ejection fraction by echocardiography. Circulation 43:480-490, 1971. 
47. FEIGENBAUM, H. Echocardiography. Philadelphia, Lea \& Febiger, 1994. cap.3, p.134-180: Echocardiographic evaluation of cardiac chambers.

48. FEIGENBAUM, H. Echocardiography. Philadelphia, Lea \& Febiger, 1994. cap.4, p.181-215: Hemodynamic information derived from echocardiography.

49. VASAN, R.S.; LARSON, M.G.; LEVY, D.; EVANS, J.C.; BENJAMIN, E.J. Distribution and categorization of echocardiographic measurements in relation to reference limits. Circulation 96:1863-1873, 1997.

50. ROSNER, B. Fundamentals of biostatistics (2.ed.). Boston, Massachusetts, PWS Publishers, 1986.

51. LEE, E. Statistical methods for survival data analysis. Belmont, CA, Life-time Learning Publications, 1980.

52. COX, D.R. Regression models and life-tables (with discussion). J.R. Stat Soc (B) 34:187-220, 1972.

53. FRANK, S.; BRAUNWALD, E. I diopathic hypertrophic subaortic stenosis - clinical analysis of 126 patients with emphasis on the natural history. Circulation 37:759788, 1968.

54. AZZANO, O.; BOZIO, A.; SASSOLAS, F.; Di FILLIPPO, S.; AGÉ, C.; ANDRÉ, M.; JOCTEUR-MONROZIER, D.; NORMAND, J. Histoire naturelle de la myocardiopathie hypertrophique obstructive du sujet jeune: à propos de 40 observations. Arch Mal Cœur 88:667-672, 1995

55. YOSHIDA, M.; NAKAMURA, Y.; FUKUHARA, T.; HIGASHIKAWA, M.; OKADA, M.; SHICHIRI, G.; KINOSHITA, M. Prognostic determinants of hypertrophic cardiomyopathy - the results of the Shiga Cardiomyopathy Study. Jpn Cir J 59:745-753, 1995.

56. FANANAPAZIR, L.; CHANG, A.C.; EPSTEIN, S.E.; McAREAVEY, D. Prognostic determinants in hypertrophic cardiomyopathy: prospective evaluation of a therapeutic strategy based on clinical, Holter, hemodynamic and 